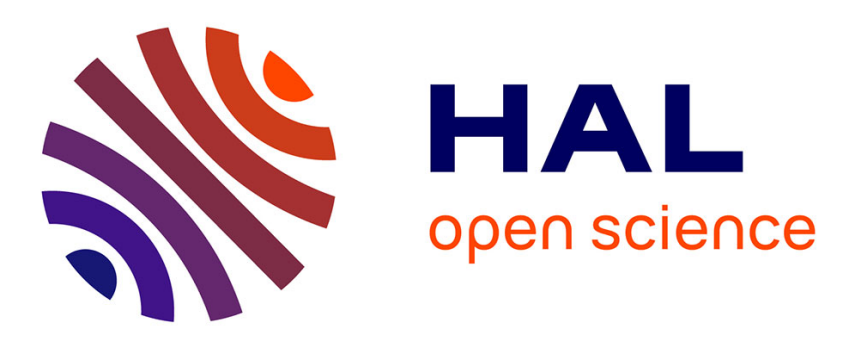

\title{
Cyclic Allyl Carbamates in Stereoselectivesyn SE' Processes: Synthetic Approach to Sarcodictyins and Eleutherobin
}

Sylvie Dhulut, Arnaud Bourin, Marie-Isabelle Lannou, Etienne Fleury, Nathalie Lensen, Evelyne Chelain, Ange Pancrazi, Janick Ardisson, Jacques Fahy

\section{To cite this version:}

Sylvie Dhulut, Arnaud Bourin, Marie-Isabelle Lannou, Etienne Fleury, Nathalie Lensen, et al.. Cyclic Allyl Carbamates in Stereoselectivesyn SE' Processes: Synthetic Approach to Sarcodictyins and Eleutherobin. European Journal of Organic Chemistry, 2007, 2007 (31), pp.5235-5243. 10.1002/ejoc.200700490 . hal-02371125

\section{HAL Id: hal-02371125 https://hal.science/hal-02371125}

Submitted on 12 Oct 2021

HAL is a multi-disciplinary open access archive for the deposit and dissemination of scientific research documents, whether they are published or not. The documents may come from teaching and research institutions in France or abroad, or from public or private research centers.
L'archive ouverte pluridisciplinaire HAL, est destinée au dépôt et à la diffusion de documents scientifiques de niveau recherche, publiés ou non, émanant des établissements d'enseignement et de recherche français ou étrangers, des laboratoires publics ou privés. 


\title{
Cyclic Allyl Carbamates in Stereoselective syn $\mathbf{S}_{\mathbf{E}}{ }^{\prime}$ Processes: Synthetic Approach to Sarcodictyins and Eleutherobin
}

\author{
Sylvie Dhulut, ${ }^{\text {[a] }}$ Arnaud Bourin, ${ }^{[\text {a] }}$ Marie-Isabelle Lannou, ${ }^{\text {[a] }}$ Etienne Fleury, ${ }^{\text {[a] }}$ \\ Nathalie Lensen, ${ }^{*[a]}$ Evelyne Chelain, ${ }^{*[a]}$ Ange Pancrazi, ${ }^{[a]}$ Janick Ardisson, ${ }^{[a]}$ and \\ Jacques Fahy*|b]
}

Keywords: Cytotoxic agents / Synthesis / $\operatorname{syn} \mathrm{S}_{\mathrm{E}}{ }^{\prime} /$ Carbamates / Terpenoids

Our synthetic approach of marine diterpenoids sarcodictyins $\mathrm{A}$ and $\mathrm{B}$ and eleutherobin relies on the one-step attachment of a C5-C9 side chain at the $\mathrm{C} 10$ position. The $\mathrm{C} 1, \mathrm{C} 10 \mathrm{cis}-$ disubstituted cyclohexene derivative is obtained in $86 \%$ yield with total stereoselectivity. The reaction is based on a syn $\mathrm{S}_{\mathrm{E}}{ }^{\prime}$ process involving a cyclic $(Z)$-allyl diisopropylcarbamate.

These compounds are potent inducers of tubulin polymerization and possess cytotoxic activity against human colon and ovarian carcinoma cell lines (IC50 10.7 and $13.7 \mathrm{~nm}$, respectively); furthermore, they are active against paclitaxel resistant tumour cell lines. ${ }^{[5]}$ Owing to the biological interest of these molecules, much work has been devoted to the total synthesis of sarcodictyins $\mathbf{1}$ and $\mathbf{2}$ and eleutherobin $\mathbf{3}$. However, to date, only two syntheses of eleutherobin $\mathbf{3}$ have been described by Nicolaou ${ }^{[6]}$ and Danishefsky, ${ }^{[7]}$ and one synthesis of sarcodictyins $\mathbf{1}$ and $\mathbf{2}$ by Nicolaou. ${ }^{[8]}$ In addition, two formal syntheses by Metz and Gennari ${ }^{[9]}$ and several approaches ${ }^{[10]}$ of these compounds were reported.

\section{Results and Discussion}

Our strategy (Scheme 2) involved the formation of the 10-membered ring B of $\mathbf{4}$ by cyclization of the conveniently substituted precursor 5 with the use of the Mukaiyama reaction.

This silyl enol ether could be constructed from diol $\mathbf{6}$, which in turn would be obtained from the key intermediate 7 by C7-C 8 the Sharpless dihydroxylation reaction, ${ }^{[11]}$ deoxygenation at $\mathrm{C}^{[12]}$ and reduction of the O-enecarbamate functionality $\left[i \mathrm{PrMgCl} /\right.$ catalytic $\left.\mathrm{Ni}(\text { acac })_{2}\right] \cdot{ }^{[13]}$

In this paper, we describe a new method for the installation of a C5-C9 side chain at the C10 position of compound 7 starting with allylic carbamate $\mathbf{8}$, which can be prepared by the vinylogous Mukaiyama aldol reaction of $(R)$-carvone (9) previously described by Gennari. ${ }^{[9 \mathrm{~b}, 10 \mathrm{a}]}$

The key step for the formation of compound 7 consisted in the $S_{E}{ }^{\prime}$ reaction of a stable allyl metal with an electrophile. More specifically, the homoaldol reaction with secondary metallated allyl carbamates reported by Hoppe was considered. ${ }^{[14-16]}$ We already demonstrated the utility of

a] Laboratoire de Synthèse Organique Sélective et Chimie Organométallique, UMR 8123, Université de Cergy-Pontoise, CNRS-UCP-ESCOM, 5 Mail Gay-Lussac Neuville 95031, Cergy-Pontoise Cedex, France E-mail: nathalie.lensen@u-cergy.fr; evelyne.chelain@u-cergy.fr

[b] Pierre Fabre Centre de Recherche en Oncologie Expérimentale, 3 rue des Satellites, 31400 Toulouse, France

E-mail: jacques.fahy@pierre-fabre.com 


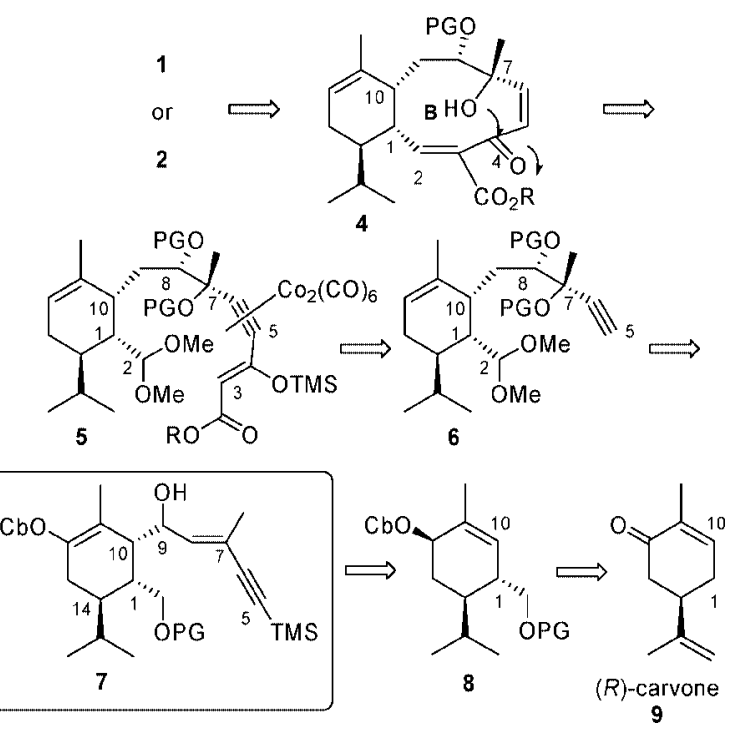

Scheme 2. Synthetic strategy.

homoaldol reactions from acyclic secondary carbamates in total syntheses, ${ }^{[17]}$ and Hoppe and coworkers described the homoaldol reaction of a $\mathrm{C} 12-\mathrm{C} 14^{[18]}$ cis-carveyl carbamate similar to 11 with acetone (Scheme 3). ${ }^{[14 b, 19]}$

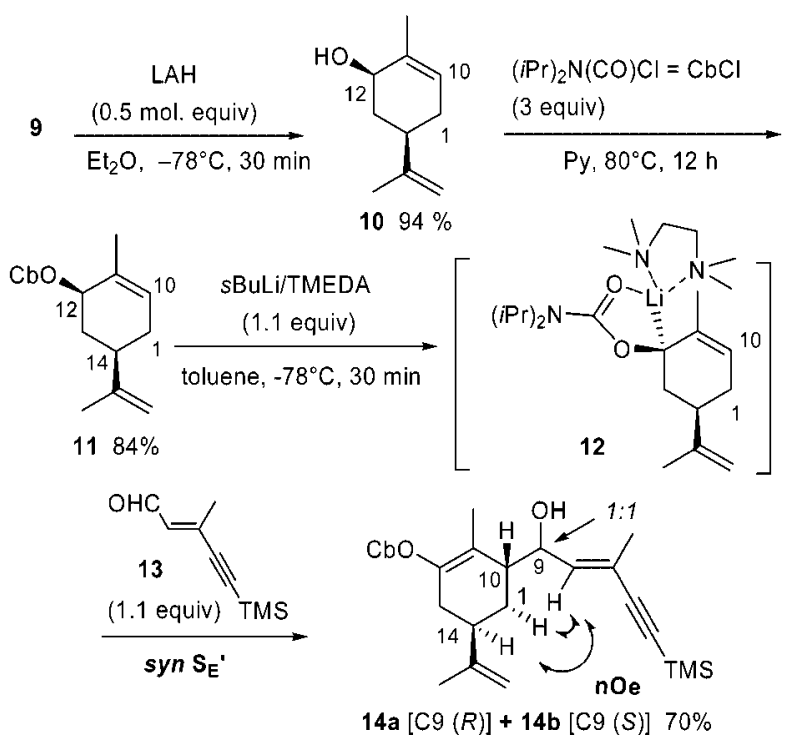

Scheme 3. Formation of C10-C14 trans carveyl alcohols 14a and 14b.

C12-C14 cis-carveyl diisopropylcarbamate $\mathbf{1 1}$ was prepared by reduction of 9 and carbamoylation and then deprotonated with $s \mathrm{BuLi} / \mathrm{TMEDA}$ (1.1 equiv., toluene, $\left.-78^{\circ} \mathrm{C}, 30 \mathrm{~min}\right)$; reaction of this lithio compound with enynal $\mathbf{1 3}^{[20]}$ delivered a 1:1 C9 diastereomeric mixture of adducts $14 \mathbf{a}$ and $14 \mathbf{b}$ in $70 \%$ yield. The configuration at the C10 centre, created with total stereoselectivity, of these two separable products was assigned from ${ }^{1} \mathrm{H}$ NMR spectroscopic analysis and nOe experiments. ${ }^{[21]}$

To improve the diastereoselectivity of this reaction at C9, a lithium/titanium exchange was then performed. Transme- tallation of lithiated $\mathbf{1 2}$ with $\mathrm{Ti}(\mathrm{O} i \mathrm{Pr})_{4}$ led to a 1:1:2 mixture of adducts 14a, 14b and $\mathbf{1 5}$ (the epimer of 14a at C10) in $30 \%$ yield (Scheme 4). ${ }^{[22]}$ However, switching to $\mathrm{TiCl}(\mathrm{O} i \mathrm{Pr})_{3}$, the $\mathrm{C} 10-\mathrm{C} 14$ cis carveyl enynol $\mathbf{1 5}$ was produced in $47 \%$ yield (along with $3 \%$ of a mixture of $\mathbf{1 4 a}$ and $\mathbf{1 4 b}$, and $31 \%$ of recovered starting material 11). ${ }^{[21]}$ Under the latter conditions, transmetallation occurred with inversion of configuration to afford the titanium intermediate $(R)$-“Ti"-12,,$^{[23]}$ and this intermediate underwent a syn $\mathrm{S}_{\mathrm{E}}{ }^{\prime}$ reaction to produce $\mathrm{C} 10-\mathrm{C} 14$ cis compound $\mathbf{1 5}$. The Re face selectivity $[(S)-C 9]$ in this case is excellent, but cannot be explained by a typical Zimmerman-Traxler transition state. The stereochemistry of the side chain at $\mathrm{C} 10$ of compound $\mathbf{1 5}$ was inferred from NMR spectroscopic analysis and nOe experiments. ${ }^{[21]}$
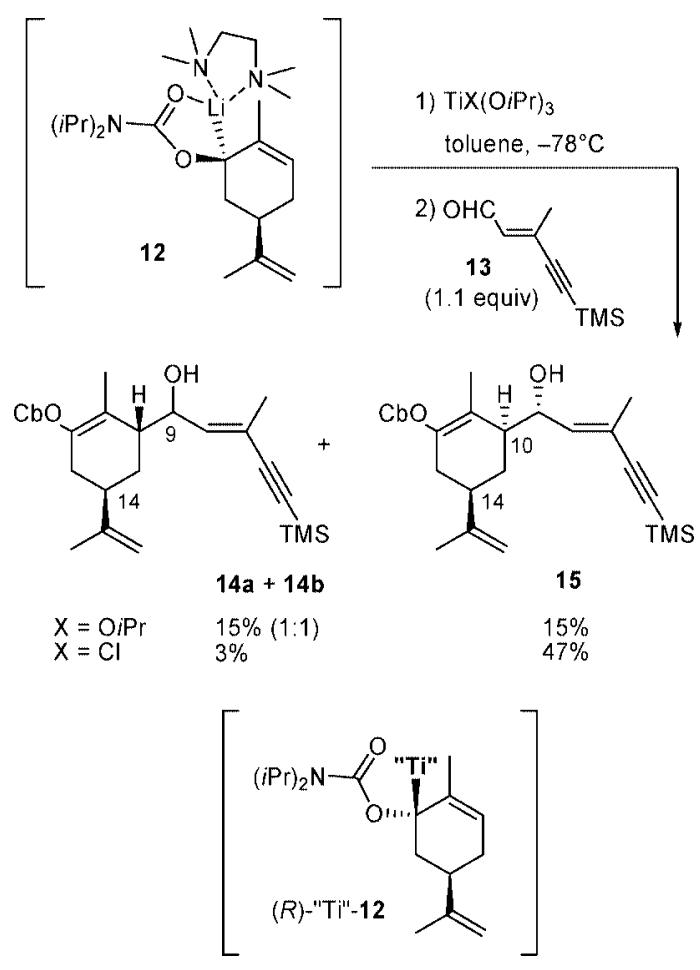

Scheme 4. Reaction between titanium carveyl $(R)$-diisopropylcarbamate $(R)$-“Ti"-12 and enynal 13.

In order to apply this method to our strategic goal, allyl carbamate 17, substituted at the $\mathrm{C} 1$ centre, was prepared from Gennari product $\mathbf{1 6}^{[\mathrm{aa}, 10 \mathrm{a}]}$ in eight steps and $29.6 \%$ overall yield from carvone (Scheme 5).

Allyl carbamate 17, when deprotonated with $s \mathrm{BuLi} /$ TMEDA ( $3.5 \mathrm{~h}$ deprotonation time instead of $30 \mathrm{~min}$ ) furnished the expected $\mathrm{C} 1-\mathrm{C} 10$ cis-disubstituted compounds 18a and $\mathbf{1 8 b}$ as a $1: 1$ mixture in $86 \%$ yield. ${ }^{[13]}$

Structural assignment of the two separated diastereomers 18a and 18b was realized by NMR spectroscopic analysis and nOe experiments of diol derivatives 19a and $\mathbf{1 9 b}$ and of ether 20, which resulted from acidic treatment (Amberlyst $15 / \mathrm{MeOH})$. Absolute configurations were deduced from NMR spectroscopic analysis of the corresponding $(R)$ and $(S)$ mandelate esters of $\mathbf{1 8 a}$ and $\mathbf{1 8 b}$. 

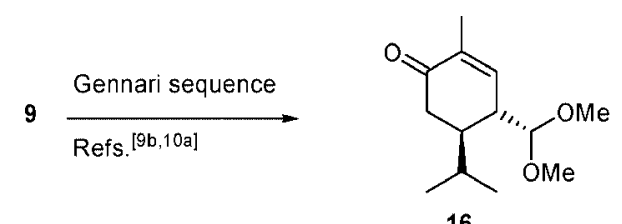

$45 \%$ for three steps ( $10 \mathrm{~g}$ scale)
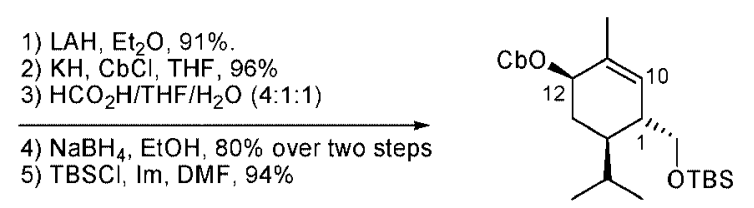

1) sBuLi/TMEDA ( 1.3 equiv.), toluene
$-78^{\circ} \mathrm{C}, 3.5 \mathrm{~h}$
2) 13 (1.3 equiv.) $86 \%$.
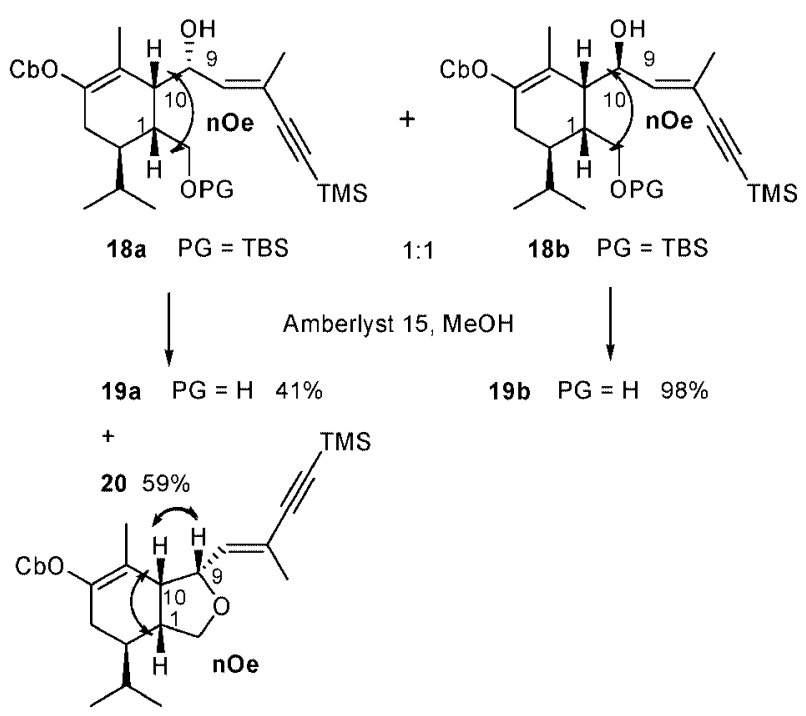

Scheme 5. Formation of the homoaldols $\mathbf{1 8 a}$ and $\mathbf{1 8 b}$.

Finally, in a more convergent synthetic approach of sarcodictyins and eleutherobin, enynal $\mathbf{2 1}^{[20]}$ was treated with 17 under the same conditions used before to deliver the two separable alcohols 22a and 22b in 57\% unoptimized yield (Scheme 6).

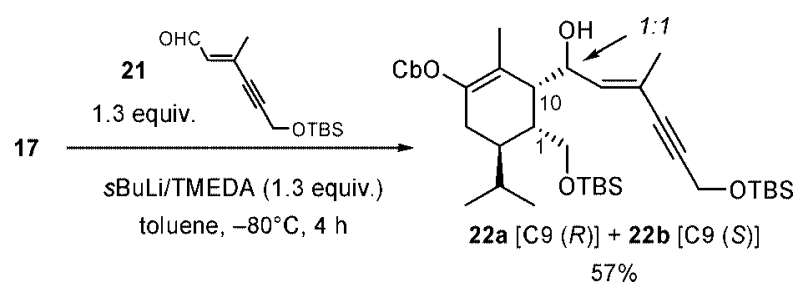

Scheme 6. Formation of the homoaldols 22a and 22b.

Further work is in progress to access the ten-membered ring $\mathrm{B}$ of sarcodictyins and eleutherobin from adducts $\mathbf{1 8 a}, \mathbf{b}$ and 22a,b.

\section{Conclusions}

We performed an efficient syn $\mathrm{S}_{\mathrm{E}}{ }^{\prime}$ reaction involving a cyclic $(Z)$ secondary allyl $\alpha$-lithiodiisopropylcarbamate. The C1,C10 cis-disubstituted precursors 18a,b and 22a,b were prepared starting from $(R)-(-)$-carvone by introduction of a side chain at $\mathrm{C} 10$ with the desired configuration. These compounds can be considered as promising intermediates in a new synthetic approach to sarcodictyins A and B and eleutherobin.

\section{Experimental Section}

General Remarks: All reactions were carried out in oven-dried or flame-dried glassware under an argon atmosphere by employing standard techniques in handling air-sensitive materials. All solvents were of reagent grade. Tetrahydrofuran (THF) and diethyl ether $\left(\mathrm{Et}_{2} \mathrm{O}\right)$ were freshly distilled from sodium/benzophenone under a nitrogen atmosphere immediately prior to use. Dichloromethane (DCM) was freshly distilled from calcium hydride. All other reagents were used as supplied. Reactions were magnetically stirred and monitored by thin layer chromatography with $0.20 \mathrm{~mm}$ SDS 60F254 precoated silica gel plates. Flash chromatography was performed with silica gel 60 (particle size $0.040-0.063 \mathrm{~mm}$ ) supplied by SDS. Yield refers to chromatographically and spectroscopically pure compounds, unless otherwise noted. Proton NMR spectra were recorded by using an internal deuterium lock at ambient temperature with a Jeol 270 or $400 \mathrm{MHz}$ spectrometer. An internal reference of $\delta_{\mathrm{H}}=7.26 \mathrm{ppm}$ was used for $\mathrm{CDCl}_{3}$. Data are represented as follows: chemical shift, multiplicity ( $\mathrm{s}=$ single, $\mathrm{d}=$ doublet, $\mathrm{t}=$ triplet, $\mathrm{q}=$ quartet), integration and coupling constant. Carbon-13 NMR spectra were recorded with a Jeol 67.8 or $100.5 \mathrm{MHz}$ spectrometer. An internal reference of $\delta_{\mathrm{C}}=77.14 \mathrm{ppm}$ was used for $\mathrm{CDCl}_{3}$. Infrared spectra were recorded with a Nicolet Impact-400.

(1R,5R)-5-Isopropenyl-2-methylcyclohex-2-enol (10): To a solution of lithium aluminium hydride (LAH; $1 \mathrm{M}, 6.7 \mathrm{~mL}, 6.7 \mathrm{mmol}$, 0.5 equiv.) at $-78^{\circ} \mathrm{C}$ was slowly added $(R)-(-)$-carvone $(9 ; 2 \mathrm{~g}$, $13.3 \mathrm{mmol})$ in $\mathrm{Et}_{2} \mathrm{O}(10 \mathrm{~mL})$. After $30 \mathrm{~min}, \mathrm{H}_{2} \mathrm{O}(450 \mu \mathrm{L}$, $25 \mathrm{mmol}), \mathrm{NaOH}(450 \mu \mathrm{L}, 25 \mathrm{mmol})$ and more $\mathrm{H}_{2} \mathrm{O}(450 \mu \mathrm{L}$, $25 \mathrm{mmol}$ ) was successively added. The solution was stirred until a white precipitate appeared, then dried with $\mathrm{MgSO}_{4}$ and filtered off. The solvent was remove under reduce pressure, and the crude residue was purified by chromatography on silica gel (cyclohexane/AcOEt, 80:20) to deliver compound $10(1.9 \mathrm{~g}, 94 \%, 95 \%$ de $)$. $[\alpha]_{\mathrm{D}}^{20}=$ $-35.5\left(c=2.28, \mathrm{CHCl}_{3}\right)\left\{\right.$ ref. $^{[14 \mathrm{~b}, 19]}[a]_{\mathrm{D}}^{20}=-35.0(c=4.00$, $\left.\left.\mathrm{CHCl}_{3}\right)\right\} .{ }^{1} \mathrm{H}$ NMR $\left(270 \mathrm{MHz}, \mathrm{CDCl}_{3}\right): \delta=5.49$ (dd, $J=2.6$, $1.3 \mathrm{~Hz}, 1 \mathrm{H}), 4.72$ (s, $2 \mathrm{H}), 4.18(\mathrm{~m}, 1 \mathrm{H}), 2.30-1.86(\mathrm{~m}, 4 \mathrm{H}), 1.75$ (s, $\left.3 \mathrm{H}, \mathrm{CH}_{3}\right), 1.74\left(\mathrm{~s}, 3 \mathrm{H}, \mathrm{CH}_{3}\right), 1.65$ (s, $\left.1 \mathrm{H}, \mathrm{OH}\right), 1.56-1.41$ (td, $J=12.2,9.9 \mathrm{~Hz}, 1 \mathrm{H}) \mathrm{ppm} .{ }^{13} \mathrm{C} \mathrm{NMR}\left(67.5 \mathrm{MHz}, \mathrm{CDCl}_{3}\right): \delta=$ $148.9(\mathrm{C}), 136.2(\mathrm{C}), 123.7(\mathrm{CH}), 109.0\left(\mathrm{CH}_{2}\right), 70.7(\mathrm{CH}), 40.4$ (CH), $37.9\left(\mathrm{CH}_{2}\right), 30.9\left(\mathrm{CH}_{2}\right), 20.5\left(\mathrm{CH}_{3}\right), 18.9\left(\mathrm{CH}_{3}\right)$ ppm. MS (GC, EI): $m / z=152 . \mathrm{C}_{10} \mathrm{H}_{16} \mathrm{O}$ (152.24): calcd. C 78.90, H 10.59; found C 79.15, H 10.68 .

(1R,4R,5R)-5-Isopropenyl-2-methylcyclohex-2-enyl Diisopropylcarbamate (11): To a solution of $\mathbf{1 0}(1.47 \mathrm{~g}, 9.6 \mathrm{mmol})$ in pyridine $(10 \mathrm{~mL})$ at $0{ }^{\circ} \mathrm{C}$ was slowly added diisopropylcarbamoyl chloride ( $4.8 \mathrm{~g}, 29 \mathrm{mmol}, 3$ equiv.). The mixture was stirred for $12 \mathrm{~h}$ at $80{ }^{\circ} \mathrm{C}$, and the mixture was then diluted at $0{ }^{\circ} \mathrm{C}$ with $\mathrm{Et}_{2} \mathrm{O}(20 \mathrm{~mL})$, $\mathrm{H}_{2} \mathrm{O}(10 \mathrm{~mL})$ and an aqueous saturated $\mathrm{NH}_{4} \mathrm{Cl}$ solution $(15 \mathrm{~mL})$. After extraction with $\mathrm{Et}_{2} \mathrm{O}$, the combined organic layers were 
washed with brine, dried with $\mathrm{MgSO}_{4}$ and filtered off. After the solvent was removed under reduced pressure, the crude residue was purified by chromatography on silica gel (cyclohexane/AcOEt, 80:20) to deliver product $11(2.25 \mathrm{~g}, 84 \%)$. $[\alpha]_{\mathrm{D}}^{20}=+0.7(c=3.0$, $\mathrm{MeOH}) .{ }^{1} \mathrm{H}$ NMR (400 MHz, $\left.\mathrm{CDCl}_{3}\right): \delta=5.52-5.51(\mathrm{~m}, 1 \mathrm{H})$, 5.47-5.37 (m, $1 \mathrm{H}), 4.68$ (s, 2 H), 4.17-3.93 (m, 1 H), 3.93-3.65 (m, $1 \mathrm{H}), 2.27$ (tdd, $J=12.3,5.2,1.6 \mathrm{~Hz}, 1 \mathrm{H}), 2.17$ (ddt, $J=12.3,6.0$, $1.4 \mathrm{~Hz}, 1 \mathrm{H}), 2.05(\mathrm{~m}, 1 \mathrm{H}), 1.95(\mathrm{~m}, 1 \mathrm{H}), 1.69\left(\mathrm{~s}, 3 \mathrm{H}, \mathrm{CH}_{3}\right), 1.64$ (s, $\left.3 \mathrm{H}, \mathrm{CH}_{3}\right), 1.47(\mathrm{td}, J=12.4,10.1 \mathrm{~Hz}, 1 \mathrm{H}), 1.19$ (s, $6 \mathrm{H}, 2$ $\left.\mathrm{CH}_{3}\right), 1.17$ (s, $\left.6 \mathrm{H}, 2 \mathrm{CH}_{3}\right)$ ppm. $\left.{ }^{13} \mathrm{C} \mathrm{NMR} \mathrm{(100} \mathrm{MHz,} \mathrm{CDCl}_{3}\right)$ : $\delta=155.6(\mathrm{C}), 148.6(\mathrm{C}), 134.2(\mathrm{C}), 125.0(\mathrm{CH}), 109.1\left(\mathrm{CH}_{2}\right), 73.1$ $(\mathrm{CH}), 46.2(\mathrm{CH}), 45.3(\mathrm{CH}), 40.5(\mathrm{CH}), 34.5\left(\mathrm{CH}_{2}\right), 30.8\left(\mathrm{CH}_{2}\right)$, $21.8\left(2 \mathrm{CH}_{3}\right), 20.6\left(\mathrm{CH}_{3}\right), 20.4\left(2 \mathrm{CH}_{3}\right), 19.2\left(\mathrm{CH}_{3}\right) \mathrm{ppm}$. IR (film): $\tilde{v}=1698,1451,1290,1160 \mathrm{~cm}^{-1}$. MS $(\mathrm{GC}, \mathrm{EI}): \mathrm{m} / \mathrm{z}=279[\mathrm{M}]^{+}$, 220, 146. $\mathrm{C}_{17} \mathrm{H}_{29} \mathrm{NO}_{2}$ (279.42): calcd. C 73.07, H 10.46, N 5.01; found C 73.14, H 10.52, N 5.05.

(2E)-3-Methyl-5-trimethylsilylpent-2-en-4-ynal (13): To a suspension of $\mathrm{Pd}(\mathrm{OAc})_{2}(180 \mathrm{mg}, 0.75 \mathrm{mmol}, 0.05$ equiv.) in THF (34 mL) at $20^{\circ} \mathrm{C}$ was added tris(2,6-dimethoxyphenyl)phosphane $(340 \mathrm{mg}$, $1.5 \mathrm{mmol}, 0.05$ equiv.). After stirring for $15 \mathrm{~min}$, ethyl butynoate (1.75 mL, $15.0 \mathrm{mmol}, 1$ equiv.) was introduced. To the resulting clear brown mixture obtained after $5 \mathrm{~min}$, was added trimethylsilylacetylene $(2.12 \mathrm{~mL}, 15.0 \mathrm{mmol}, 1$ equiv.). The reaction mixture became dark brown. After $2 \mathrm{~h}$, the mixture was concentrated under reduced pressure, and the crude residue was purified by chromatography on silica gel (cyclohexane/AcOEt, 95:5) to deliver ethyl (2E)3-methyl-5-trimethylsilylpent-2-en-4-ynoate (3.0 g, 95\%). ${ }^{1} \mathrm{H}$ NMR $\left(270 \mathrm{MHz}, \mathrm{CDCl}_{3}\right): \delta=6.04(\mathrm{~s}, 1 \mathrm{H}), 4.11(\mathrm{q}, J=7.1 \mathrm{~Hz}, 2 \mathrm{H})$, $2.22\left(\mathrm{~s}, 3 \mathrm{H}, \mathrm{CH}_{3}\right), 1.22\left(\mathrm{t}, J=7.1 \mathrm{~Hz}, 3 \mathrm{H}, \mathrm{CH}_{3}\right), 0.15(\mathrm{~s}, 9 \mathrm{H}, 3$ $\left.\mathrm{CH}_{3}\right)$ ppm. ${ }^{13} \mathrm{C}$ NMR $\left(67.5 \mathrm{MHz}, \mathrm{CDCl}_{3}\right): \delta=166.3(\mathrm{C}), 137.8$ (C), $125.3(\mathrm{CH}), 106.8(\mathrm{C}), 99.4(\mathrm{C}), 60.3\left(\mathrm{CH}_{2}\right), 19.9\left(\mathrm{CH}_{3}\right), 14.5$ $\left(\mathrm{CH}_{3}\right), 0.0\left(3 \mathrm{CH}_{3}\right)$ ppm. MS $\left(\mathrm{GC}, \mathrm{CI}, \mathrm{CH}_{4}\right): m / z=211[\mathrm{M}+\mathrm{H}]^{+}$.

To a solution of ethyl (2E)-3-methyl-5-trimethylsilyl pent-2-ene-4ynoate $(2.4 \mathrm{~g}, 11.5 \mathrm{mmol})$ in $\mathrm{CH}_{2} \mathrm{Cl}_{2}(24.6 \mathrm{~mL})$ at $-78^{\circ} \mathrm{C}$ was added dropwise a solution of DIBAL-H $\left(1 \mathrm{M}\right.$ in $\mathrm{CH}_{2} \mathrm{Cl}_{2}, 31 \mathrm{~mL}$, $31 \mathrm{mmol}, 2.7$ equiv.). After $2 \mathrm{~h}$ at $-78^{\circ} \mathrm{C}$, to the reaction mixture was added ethyl acetate then potassium tartrate $(46 \mathrm{mmol}$, 4 equiv.), and the mixture was stirred for $1 \mathrm{~h}$. The aqueous phase was extracted with $\mathrm{CH}_{2} \mathrm{Cl}_{2}$, and the combined organic layers were washed with brine, dried with $\mathrm{MgSO}_{4}$ and filtered off. After the solvent was removed under reduced pressure, the crude residue was purified by chromatography on silica gel (cyclohexane/AcOEt, $80: 20)$ to deliver the (2E)-3-methyl-5-trimethylsilyl pent-2-en-4-ynol (1.65 g, 86\%). ${ }^{1} \mathrm{H}$ NMR $\left(270 \mathrm{MHz}, \mathrm{CDCl}_{3}\right): \delta=6.05(\mathrm{t}, J=6.6 \mathrm{~Hz}$, $1 \mathrm{H}), 4.19(\mathrm{~d}, J=6.6 \mathrm{~Hz}, 2 \mathrm{H}), 1.81\left(\mathrm{~s}, 3 \mathrm{H}, \mathrm{CH}_{3}\right), 1.67(\mathrm{~s}, 1 \mathrm{H}$, $\mathrm{OH}), 0.17$ (s, $\left.9 \mathrm{H}, 3 \mathrm{CH}_{3}\right)$ ppm. ${ }^{13} \mathrm{C} \mathrm{NMR}\left(67.5 \mathrm{MHz}, \mathrm{CDCl}_{3}\right): \delta$ $=136.6(\mathrm{CH}), 120.7(\mathrm{C}), 107.4(\mathrm{C}), 92.2(\mathrm{C}), 59.1\left(\mathrm{CH}_{2}\right), 17.4$ $\left(\mathrm{CH}_{3}\right), 0.0\left(3 \mathrm{CH}_{3}\right)$ ppm. IR (film): $\tilde{v}=3339,2960,2146,1634$, $1445,1245,1009 \mathrm{~cm}^{-1}$. MS (GC, EI): $m / z=168$.

IBX $^{[24]}$ (734 mg, $2.62 \mathrm{mmol}, 2.2$ equiv.) was dissolved in DMSO $(5 \mathrm{~mL})$ at $20^{\circ} \mathrm{C}$. When the mixture became transparent, a DMSO solution $(3 \mathrm{~mL})$ of $(2 E)$-3-methyl-5-trimethylsilyl pent-2-en-4-ynol (200 mg, $1.19 \mathrm{mmol}$ ) was added. After stirring for $2 \mathrm{~h}$ at $20^{\circ} \mathrm{C}$, $\mathrm{H}_{2} \mathrm{O}$ was added at $0{ }^{\circ} \mathrm{C}$, and the reaction mixture was filtered through a short pad of celite. After extraction with $\mathrm{Et}_{2} \mathrm{O}$, the combined organic layers were washed with brine, dried with $\mathrm{MgSO}_{4}$ and filtered off, and the solvents were removed under reduced pressure. Crude aldehyde 13 (195 mg) was used without further purification in the next step. ${ }^{1} \mathrm{H}$ NMR $\left(400 \mathrm{MHz}, \mathrm{CDCl}_{3}\right): \delta=9.95$ (d, $J=8.2 \mathrm{~Hz}, 1 \mathrm{H}), 6.13(\mathrm{dq}, J=8.2,1.4 \mathrm{~Hz}, 1 \mathrm{H}), 2.21(\mathrm{~d}, J=$ $\left.1.4 \mathrm{~Hz}, 3 \mathrm{H}, \mathrm{CH}_{3}\right),-0.15\left(\mathrm{~s}, 9 \mathrm{H}, 3 \mathrm{CH}_{3}\right) \mathrm{ppm} .{ }^{13} \mathrm{C} \mathrm{NMR}$ $\left(100 \mathrm{MHz}, \mathrm{CDCl}_{3}\right): \delta=190.2(\mathrm{CH}), 140.0(\mathrm{C}), 134.0(\mathrm{CH}), 105.5$
(C), $105.0(\mathrm{C}), 18.3\left(\mathrm{CH}_{3}\right),-0.5\left(3 \mathrm{CH}_{3}\right) \mathrm{ppm}$. MS (GC, EI): $\mathrm{m} / \mathrm{z}$ $=166[\mathrm{M}]^{+}$.

\section{Reaction of Carbamate 11 with Enynal 13 Under sBuLi/TMEDA/} toluene Conditions

[3R(1R,2E),5R]-3-[1-Hydroxy-3-methyl-5-(trimethylsilyl)pent-2-en4-ynyl]-2-methyl-5-(prop-1-en-2-yl)cyclohex-1-enyl Diisopropylcarbamate $(14 a)$ and $[3 R(1 S, 2 E), 5 R]-3-[1-H y d r o x y-3-m e t h y l-5-(t r i-$ methylsilyl)pent-2-en-4-ynyl]-2-methyl-5-(prop-1-en-2-yl)cyclohex-1enyl Diisopropylcarbamate (14b): To a solution of carbamate 11 (500 mg, $1.8 \mathrm{mmol})$ and TMEDA ( $300 \mu \mathrm{L}, 2 \mathrm{mmol}, 1.1$ equiv.) in toluene $(10 \mathrm{~mL})$ at $-78^{\circ} \mathrm{C}$ was slowly added $s \mathrm{BuLi}(1.28 \mathrm{M}$ in hexanes, $1.5 \mathrm{~mL}, 2 \mathrm{mmol}, 1.1$ equiv.). The clear reaction mixture became deep yellow. After $30 \mathrm{~min}$ deprotonation time at $-78{ }^{\circ} \mathrm{C}$, a solution of aldehyde 13 (330 mg, $2 \mathrm{mmol}, 1.1$ equiv.) in toluene $(5 \mathrm{~mL})$ was added. After stirring for $1 \mathrm{~h}$ at $-78^{\circ} \mathrm{C}$ the reaction was quenched by the addition of a $\mathrm{MeOH} / \mathrm{H}_{2} \mathrm{O} / \mathrm{HCl}(1 \mathrm{~N})(10 \mathrm{~mL} /$ $4 \mathrm{~mL} / 1 \mathrm{~mL}$ ) mixture. The temperature was warmed to $20^{\circ} \mathrm{C}$, and the mixture was extracted with $\mathrm{Et}_{2} \mathrm{O}$. The combined organic layers were washed with brine, dried with $\mathrm{MgSO}_{4}$ and filtered off. After the solvent was removed under reduced pressure, the crude residue was purified by chromatography on silica gel (cyclohexane/AcOEt, 90:10) to deliver title products $\mathbf{1 4 a}(285 \mathrm{mg})$ and $\mathbf{1 4 b}(280 \mathrm{mg})$ in an overall yield of $70 \%(565 \mathrm{mg}) ; \mathbf{1 4 a} / \mathbf{1 4 b}, 1: 1$. Data for $\mathbf{1 4 a}:[\alpha]_{\mathrm{D}}^{20}$ $=+3.1\left(c=1.08, \mathrm{CHCl}_{3}\right) .{ }^{1} \mathrm{H}$ NMR $\left(400 \mathrm{MHz}, \mathrm{CDCl}_{3}\right): \delta=6.04$ $(\mathrm{dq}, J=7.8,1.0 \mathrm{~Hz}, 1 \mathrm{H}), 4.74(\mathrm{~d}, J=0.5 \mathrm{~Hz}, 1 \mathrm{H}), 4.72(\mathrm{~d}, J=$ $0.5 \mathrm{~Hz}, 1 \mathrm{H}), 4.67(\mathrm{dd}, J=7.8,3.7 \mathrm{~Hz}, 1 \mathrm{H}), 4.08(\mathrm{~m}, 1 \mathrm{H}), 3.81$ $(\mathrm{m}, 1 \mathrm{H}), 2.94(\mathrm{~m}, 1 \mathrm{H}), 2.26(\mathrm{~m}$ sharp, $1 \mathrm{H}), 2.18(\mathrm{~m}, 1 \mathrm{H}), 2.08$ $(\mathrm{m}, 1 \mathrm{H}), 1.98(\mathrm{~m}, 1 \mathrm{H}), 1.85\left(\mathrm{~d}, J=1.4 \mathrm{~Hz}, 3 \mathrm{H}, \mathrm{CH}_{3}\right), 1.75(\mathrm{~s}, 3$ $\left.\mathrm{H}, \mathrm{CH}_{3}\right), 1.63\left(\mathrm{~s}, 3 \mathrm{H}, \mathrm{CH}_{3}\right), 1.52(\mathrm{td}, J=13.3,6.0 \mathrm{~Hz}, 1 \mathrm{H}), 1.26$ (s, $\left.6 \mathrm{H}, 2 \mathrm{CH}_{3}\right), 1.20\left(\mathrm{~s}, 6 \mathrm{H}, 2 \mathrm{CH}_{3}\right), 0.18\left(\mathrm{~s}, 9 \mathrm{H}, 3 \mathrm{CH}_{3}\right) \mathrm{ppm}$. ${ }^{13} \mathrm{C}$ NMR (100 MHz, $\mathrm{CDCl}_{3}$ ): $\delta=153.9(\mathrm{C}), 149.3$ (C), 145.7 (C), $139.6(\mathrm{CH}), 119.5(\mathrm{C}), 119.5(\mathrm{C}), 109.3\left(\mathrm{CH}_{2}\right), 108.1(\mathrm{C}), 91.6(\mathrm{C})$, $69.3(\mathrm{CH}), 47.2(\mathrm{CH}), 46.0(\mathrm{CH}), 45.4(\mathrm{CH}), 38.2(\mathrm{CH}), 32.7\left(\mathrm{CH}_{2}\right)$, $28.2\left(\mathrm{CH}_{2}\right), 21.7\left(2 \mathrm{CH}_{3}\right), 20.7\left(\mathrm{CH}_{3}\right), 20.6\left(2 \mathrm{CH}_{3}\right), 18.2\left(\mathrm{CH}_{3}\right)$, $14.8\left(\mathrm{CH}_{3}\right), 0.4\left(3 \mathrm{CH}_{3}\right) \mathrm{ppm}$. IR (film): $\tilde{v}=3421,2966,2141,1680$, $1445,1296,1148 \mathrm{~cm}^{-1}$. MS (GC, CI, $\left.\mathrm{CH}_{4}\right): m / z=446[\mathrm{M}+\mathrm{H}]^{+}$, 279, 128, 109, 86, 73. $\mathrm{C}_{26} \mathrm{H}_{43} \mathrm{NO}_{3} \mathrm{Si}$ (445.72): calcd. C 70.06, $\mathrm{H}$ 9.72, N 3.14; found C 70.14, H 9.77, N 3.18. Data for 14b: $[\alpha]_{\mathrm{D}}^{20}=$ $-4.9\left(c=0.95, \mathrm{CHCl}_{3}\right) .{ }^{1} \mathrm{H} \mathrm{NMR}\left(400 \mathrm{MHz}, \mathrm{CDCl}_{3}\right): \delta=5.94(\mathrm{dq}$, $J=9.2,1.4 \mathrm{~Hz}, 1 \mathrm{H}), 4.78-4.70(\mathrm{~m}, 2 \mathrm{H}), 4.49(\mathrm{dd}, J=9.2,5.5 \mathrm{~Hz}$, $1 \mathrm{H}), 4.15-4.00(\mathrm{~m}, 1 \mathrm{H}), 3.92-3.72(\mathrm{~m}, 1 \mathrm{H}), 2.72-2.60(\mathrm{~m}, 1 \mathrm{H})$, 2.37-2.31 (m, 1 H), 2.20-2.04 (m, $2 \mathrm{H}), 1.82\left(\mathrm{~s}, 3 \mathrm{H}, \mathrm{CH}_{3}\right), 1.72-$ $1.58(\mathrm{~m}, 2 \mathrm{H}), 1.71\left(\mathrm{~s}, 3 \mathrm{H}, \mathrm{CH}_{3}\right), 1.69\left(\mathrm{~s}, 3 \mathrm{H}, \mathrm{CH}_{3}\right), 1.32-1.19(\mathrm{~m}$, $\left.12 \mathrm{H}, 4 \mathrm{CH}_{3}\right), 0.12\left(\mathrm{~s}, 9 \mathrm{H}, 3 \mathrm{CH}_{3}\right) \mathrm{ppm} .{ }^{13} \mathrm{C} \mathrm{NMR}(100 \mathrm{MHz}$, $\left.\mathrm{CDCl}_{3}\right): \delta=153.8(\mathrm{C}), 149.0(\mathrm{C}), 144.9(\mathrm{C}), 141.5(\mathrm{CH}), 120.4(\mathrm{C})$, $119.7(\mathrm{C}), 109.7\left(\mathrm{CH}_{2}\right), 108.0(\mathrm{C}), 92.1(\mathrm{C}), 72.7(\mathrm{CH}), 47.1(\mathrm{CH})$, $46.0(\mathrm{CH}), 45.9(\mathrm{CH}), 38.2(\mathrm{CH}), 33.0\left(\mathrm{CH}_{2}\right), 31.5\left(\mathrm{CH}_{2}\right), 21.8(2$ $\left.\mathrm{CH}_{3}\right), 20.8\left(2 \mathrm{CH}_{3}\right), 20.7\left(\mathrm{CH}_{3}\right), 18.1\left(\mathrm{CH}_{3}\right), 17.3\left(\mathrm{CH}_{3}\right), 0.3(3$ $\left.\mathrm{CH}_{3}\right)$ ppm. MS (GC, CI, $\left.\mathrm{CH}_{4}\right): m / z=446[\mathrm{M}+\mathrm{H}]^{+}, 279,128,109$, 86, 73. $\mathrm{C}_{26} \mathrm{H}_{43} \mathrm{NO}_{3} \mathrm{Si}$ (445.72): calcd. C 70.06, H 9.72, N 3.14; found C 70.14, H 9.77, N 3.18.

(R)-Mandelate Ester of 14a: ${ }^{1} \mathrm{H}$ NMR $\left(400 \mathrm{MHz}, \mathrm{CDCl}_{3}\right): \delta=$ $7.38-7.28(\mathrm{~m}, 5 \mathrm{H}), 5.89(\mathrm{dq}, J=9.2,1.4 \mathrm{~Hz}, 1 \mathrm{H}), 5.85(\mathrm{dd}, J=$ 9.2, 4.6 Hz, 1 H), $4.67(\mathrm{~s}, 1 \mathrm{H}), 4.64(\mathrm{~s}, 1 \mathrm{H}), 4.59$ (s, $1 \mathrm{H}), 4.05-$ $3.90(\mathrm{~m}, 1 \mathrm{H}), 3.90-3.75(\mathrm{~m}, 1 \mathrm{H}), 3.36\left(\mathrm{~s}, 3 \mathrm{H}, \mathrm{CH}_{3}\right), 2.34-2.25$ $(\mathrm{m}, 1 \mathrm{H}), 2.28-2.23(\mathrm{~m}, 1 \mathrm{H}), 2.05-1.98(\mathrm{~m}, 1 \mathrm{H}), 1.89$ (d, $J=$ $\left.1.4 \mathrm{~Hz}, 3 \mathrm{H}, \mathrm{CH}_{3}\right), 1.85-1.60(\mathrm{~m}, 2 \mathrm{H}), 1.57\left(\mathrm{~s}, 3 \mathrm{H}, \mathrm{CH}_{3}\right), 1.51(\mathrm{~s}$, $\left.3 \mathrm{H}, \mathrm{CH}_{3}\right), 1.42(\mathrm{td}, J=13.3 ; 6.4 \mathrm{~Hz}, 1 \mathrm{H}), 1.3-1.15(\mathrm{~m}, 12 \mathrm{H}, 4$ $\left.\mathrm{CH}_{3}\right), 0.16\left(\mathrm{~s}, 9 \mathrm{H}, 3 \mathrm{CH}_{3}\right)$ ppm.

(S)-Mandelate Ester of 14a: ${ }^{1} \mathrm{H}$ NMR (400 MHz, $\left.\mathrm{CDCl}_{3}\right): \delta=$ $7.40-7.28(\mathrm{~m}, 5 \mathrm{H}), 5.82(\mathrm{dd}, J=9.2,4.6 \mathrm{~Hz}, 1 \mathrm{H}), 5.72(\mathrm{dq}, J=$ 9.2, $1.4 \mathrm{~Hz}, 1 \mathrm{H}), 4.80(\mathrm{~s}, 1 \mathrm{H}), 4.71(\mathrm{~s}, 1 \mathrm{H}), 4.70$ (s, $1 \mathrm{H}), 4.1-$ 
$3.92(\mathrm{~m}, 1 \mathrm{H}), 3.92-3.74(\mathrm{~m}, 1 \mathrm{H}), 3.40(\mathrm{~s}, 3 \mathrm{H}), 2.70-2.59(\mathrm{~m}, 1$ H), 2.38-2.32 (m, 1 H), 2.16-2.10 (m, $1 \mathrm{H}), 1.9-1.76(\mathrm{~m}, 1 \mathrm{H}), 1.81$ (d, $\left.J=1.4 \mathrm{~Hz}, 3 \mathrm{H}, \mathrm{CH}_{3}\right), 1.68\left(\mathrm{~s}, 3 \mathrm{H}, \mathrm{CH}_{3}\right), 1.58\left(\mathrm{~s}, 3 \mathrm{H}, \mathrm{CH}_{3}\right)$, $1.55(\mathrm{td}, J=13.3,6.4 \mathrm{~Hz}, 1 \mathrm{H}), 1.3-1.16\left(\mathrm{~m}, 12 \mathrm{H}, 4 \mathrm{CH}_{3}\right), 0.16$ (s, $\left.9 \mathrm{H}, 3 \mathrm{CH}_{3}\right)$ ppm.

\section{Reaction of Carbamate 11 with Enynal 13 under $s$ BuLi/TMEDA/ Toluene/Ti(OiPr) ${ }_{4}$ Conditions}

[3R(1R,2E),5R]-3-[1-Hydroxy-3-methyl-5-(trimethylsilyl)pent-2-en4-ynyl]-2-methyl-5-(prop-1-en-2-yl)cyclohex-1-enyl Diisopropylcarbamate (14a), [3R(1S,2E),5R]-3-[1-Hydroxy-3-methyl-5-(trimethylsilyl)pent-2-en-4-ynyl]-2-methyl-5-(prop-1-en-2-yl)cyclohex-1-enyl Diisopropylcarbamate (14b) and [3S(1R,2E),5R]-3-[1-Hydroxy-3methyl-5-(trimethylsilyl)pent-2-en-4-ynyl]-2-methyl-5-(prop-1-en-2yl)cyclohex-1-enyl Diisopropylcarbamate (15): To a solution of carbamate 11 (137 mg, $0.49 \mathrm{mmol})$ and TMEDA $(170 \mu \mathrm{L}, 0.54 \mathrm{mmol}$, 1.1 equiv. ) in toluene $(4 \mathrm{~mL})$ at $-78^{\circ} \mathrm{C}$ was slowly added $s \mathrm{BuLi}$ (1.2 $\mathrm{M}$ in hexanes, $82 \mu \mathrm{L}, 0.54 \mathrm{mmol}, 1.1$ equiv.). The clear reaction mixture became deep yellow. After $30 \mathrm{~min}$ deprotonation time at $-78^{\circ} \mathrm{C}$, a solution of $\mathrm{Ti}(\mathrm{O} i \mathrm{Pr})_{4}(432 \mu \mathrm{L}, 1.5 \mathrm{mmol}, 3$ equiv. $)$ in toluene $(1 \mathrm{~mL})$ was added at $-78{ }^{\circ} \mathrm{C}$, and the reaction mixture became brown green. Stirring was maintained for $30 \mathrm{~min}$ at $-78^{\circ} \mathrm{C}$ before the addition of a solution of aldehyde $13(90 \mathrm{mg}, 0.54 \mathrm{mmol}$, 1.1 equiv.) in toluene $(1.5 \mathrm{~mL})$. The orange-coloured reaction mixture was stirred at $-78{ }^{\circ} \mathrm{C}$ for $1 \mathrm{~h}$ and diluted at $0{ }^{\circ} \mathrm{C}$ with $\mathrm{Et}_{2} \mathrm{O}$ and an aqueous saturated $\mathrm{NH}_{4} \mathrm{Cl}$ solution. The aqueous phase was extracted with $\mathrm{Et}_{2} \mathrm{O}$, and the combined organic layers were washed with brine, dried with $\mathrm{MgSO}_{4}$ and filtered off. After removal of the solvents under reduced pressure, the crude residue was purified by chromatography on silica gel (cyclohexane/AcOEt, 80:20) to deliver starting material 11 (41 mg, 30\%), title compounds 14a and 14b (35 mg, 16\%; 14a/14b, 1:1) and $\mathbf{1 5}(33 \mathrm{mg}, 15 \%$, see below for description).

Reaction of Carbamate 11 with Enynal 13 under sBuLi/TMEDA/ Toluene/TiCl(OiPr $)_{3}$ Conditions

[3S $(1 R, 2 E), 5 R]-3-[1-H y d r o x y-3-m e t h y l-5-(t r i m e t h y l s i l y l) p e n t-2-e n-$ 4-ynyll-2-methyl-5-(prop-1-en-2-yl)cyclohex-1-enyl Diisopropylcarbamate (15): To a solution of carbamate 11 (305 mg, $1.09 \mathrm{mmol})$ and TMEDA (182 $\mu \mathrm{L}, 1.2 \mathrm{mmol}, 1.1$ equiv.) in toluene $(4.5 \mathrm{~mL})$ at $-78{ }^{\circ} \mathrm{C}$ was slowly added $s \mathrm{BuLi}(1.3 \mathrm{~m}$ in hexanes, $923 \mu \mathrm{L}$, $1.2 \mathrm{mmol}, 1.1$ equiv.). The clear reaction mixture became deep yellow. After 30 min deprotonation time at $-78^{\circ} \mathrm{C}$, a solution of $\mathrm{TiCl}(\mathrm{O} i \mathrm{Pr})_{3}(781 \mu \mathrm{L}, 3.27 \mathrm{mmol}, 3$ equiv. $)$ in toluene $(1.5 \mathrm{~mL})$ was added at $-78^{\circ} \mathrm{C}$, and the reaction mixture became brown green. Stirring was maintained for $20 \mathrm{~min}$ at $-78{ }^{\circ} \mathrm{C}$ before the addition of a solution of aldehyde 13 (200 mg, $1.2 \mathrm{mmol}, 1.1$ equiv.) in toluene $(2.5 \mathrm{~mL})$. The orange-coloured reaction mixture was stirred at $-78^{\circ} \mathrm{C}$ for $1 \mathrm{~h}$ and diluted at $0{ }^{\circ} \mathrm{C}$ with $\mathrm{Et}_{2} \mathrm{O}$ and an aqueous saturated $\mathrm{NH}_{4} \mathrm{Cl}$ solution. The aqueous phase was extracted with $\mathrm{Et}_{2} \mathrm{O}$, and the combined organic layers were washed with brine, dried with $\mathrm{MgSO}_{4}$ and filtered off. After the solvents were removed under reduced pressure, the crude residue was purified by chromatography on silica gel (cyclohexane/AcOEt, 80:20) to deliver compound 15 (367 mg, 47\%) together with starting material 11 (241 mg, 31\%) and 14a and 14b (25 mg, 3\%; 14a/14b, 1:1). Data for 15: $[a]_{\mathrm{D}}^{20}=+21.7\left(c=2.0, \mathrm{CHCl}_{3}\right) .{ }^{1} \mathrm{H} \mathrm{NMR}(400 \mathrm{MHz}$, $\left.\mathrm{CDCl}_{3}\right): \delta=6.02(\mathrm{dq}, J=8.2,1.4 \mathrm{~Hz}, 1 \mathrm{H}), 4.75$ (s, $\left.2 \mathrm{H}\right), 4.59$ (dd, $J=8.2,4.2 \mathrm{~Hz}, 1 \mathrm{H}), 3.99$ (m, $1 \mathrm{H}), 3.87$ (m, $1 \mathrm{H}), 2.68(\mathrm{~m}, 1 \mathrm{H})$, $2.35(\mathrm{~m}, 1 \mathrm{H}), 2.18(\mathrm{tq}, J=14.0,2.5 \mathrm{~Hz}, 1 \mathrm{H}), 2.14(\mathrm{~m}, 1 \mathrm{H}), 1.97$ (ddt, $J=13.3,6.3,2.5 \mathrm{~Hz}, 1 \mathrm{H}), 1.90\left(\mathrm{~d}, J=1.4 \mathrm{~Hz}, 3 \mathrm{H}, \mathrm{CH}_{3}\right)$, $1.75\left(\mathrm{~s}, 3 \mathrm{H}, \mathrm{CH}_{3}\right), 1.55\left(\mathrm{~s}, 3 \mathrm{H}, \mathrm{CH}_{3}\right), 1.42(\mathrm{~s}, 1 \mathrm{H}, \mathrm{OH}), 1.37$ (q, $J=13.3 \mathrm{~Hz}, 1 \mathrm{H}), 1.26\left(\mathrm{~m}, 6 \mathrm{H}, 2 \mathrm{CH}_{3}\right), 1.24\left(\mathrm{~m}, 6 \mathrm{H}, 2 \mathrm{CH}_{3}\right)$, 0.19 (s, $\left.9 \mathrm{H}, 3 \mathrm{CH}_{3}\right)$ ppm. ${ }^{13} \mathrm{C} \mathrm{NMR}\left(100 \mathrm{MHz}, \mathrm{CDCl}_{3}\right): \delta=153.1$
(C), 148.7 (C), 144.7 (C), $137.8(\mathrm{CH}), 121.7$ (C), 119.1 (C), 109.3 $\left(\mathrm{CH}_{2}\right), 107.7(\mathrm{C}), 92.3(\mathrm{C}), 70.0(\mathrm{CH}), 46.5(\mathrm{CH}), 45.8(\mathrm{CH}), 45.2$ $(\mathrm{CH}), 40.9(\mathrm{CH}), 33.3\left(\mathrm{CH}_{2}\right), 30.0\left(\mathrm{CH}_{2}\right), 21.8\left(2 \mathrm{CH}_{3}\right), 20.7\left(\mathrm{CH}_{3}\right)$, $20.6\left(\mathrm{CH}_{3}\right), 20.4\left(\mathrm{CH}_{3}\right), 17.9\left(\mathrm{CH}_{3}\right), 13.9\left(\mathrm{CH}_{3}\right),-0.02\left(3 \mathrm{CH}_{3}\right)$ ppm. IR (film): $\tilde{v}=3402,2929,1653,1409,1249,1211,1044,1010$, $841,800,744 \mathrm{~cm}^{-1}$. MS $\left(\mathrm{GC}, \mathrm{CI}, \mathrm{CH}_{4}\right): \mathrm{m} / z=446[\mathrm{M}+\mathrm{H}]^{+}$. $\mathrm{C}_{26} \mathrm{H}_{43} \mathrm{NO}_{3} \mathrm{Si}$ (445.72): calcd. C 70.06, $\mathrm{H}$ 9.72, $\mathrm{N} \mathrm{3.14}$; found $\mathrm{C}$ $70.12, \mathrm{H} 9.75, \mathrm{~N} 3.18$.

(R)-Mandelate Ester of 15: ${ }^{1} \mathrm{H}$ NMR (400 MHz, $\left.\mathrm{CDCl}_{3}\right): \delta=7.45$ $7.25(\mathrm{~m}, 5 \mathrm{H}), 5.86(\mathrm{dq}, J=8.2,1.4 \mathrm{~Hz}, 1 \mathrm{H}), 5.81(\mathrm{dd}, J=8.2$, $4.2 \mathrm{~Hz}, 1 \mathrm{H}), 4.71(\mathrm{~s}, 1 \mathrm{H}), 4.67(\mathrm{~s}, 1 \mathrm{H}), 4.59$ (s, $1 \mathrm{H}), 3.97$ (m, 1 H), $3.84(\mathrm{~m}, 1 \mathrm{H}), 3.41(\mathrm{~s}, 3 \mathrm{H}), 2.50(\mathrm{~m}, 1 \mathrm{H}), 2.08(\mathrm{~m}, 1 \mathrm{H}), 1.92$ $(\mathrm{m}, 1 \mathrm{H}), 1.86\left(\mathrm{~d}, J=1.4 \mathrm{~Hz}, 3 \mathrm{H}, \mathrm{CH}_{3}\right), 1.70(\mathrm{~m}, 1 \mathrm{H}), 1.59(\mathrm{~s}, 3$ $\left.\mathrm{H}, \mathrm{CH}_{3}\right), 1.41\left(\mathrm{~s}, 3 \mathrm{H}, \mathrm{CH}_{3}\right), 1.30-1.27(\mathrm{~m}, 1 \mathrm{H}), 1.24(\mathrm{~m}, 6 \mathrm{H}, 2$ $\left.\mathrm{CH}_{3}\right), 1.22\left(\mathrm{~m}, 6 \mathrm{H}, 2 \mathrm{CH}_{3}\right), 1.10-1.00(\mathrm{~m}, 1 \mathrm{H}), 0.19(\mathrm{~s}, 9 \mathrm{H}, 3$ $\left.\mathrm{CH}_{3}\right) \mathrm{ppm}$.

(S)-Mandelate Ester of 15: ${ }^{1} \mathrm{H}$ NMR (400 MHz, $\left.\mathrm{CDCl}_{3}\right): \delta=7.43$ $7.28(\mathrm{~m}, 5 \mathrm{H}), 5.76(\mathrm{dd}, J=8.7,5.0 \mathrm{~Hz}, 1 \mathrm{H}), 5.72(\mathrm{dq}, J=8.7$, $1.4 \mathrm{~Hz}, 1 \mathrm{H}), 5.01(\mathrm{~s}, 1 \mathrm{H}), 4.74(\mathrm{~s}, 1 \mathrm{H}), 4.72(\mathrm{~s}, 1 \mathrm{H}), 3.97$ (m, 1 H), 3.85 (m, $1 \mathrm{H}), 3.39$ (s, $3 \mathrm{H}), 2.77(\mathrm{~m}, 1 \mathrm{H}), 2.29(\mathrm{~m}, 1 \mathrm{H}), 2.13$ $(\mathrm{m}, 1 \mathrm{H}), 1.88(\mathrm{~m}, 1 \mathrm{H}), 1.72\left(\mathrm{~s}, 3 \mathrm{H}, \mathrm{CH}_{3}\right), 1.56(\mathrm{~d}, J=1.4 \mathrm{~Hz}, 3$ $\left.\mathrm{H}, \mathrm{CH}_{3}\right), 1.47\left(\mathrm{~s}, 3 \mathrm{H}, \mathrm{CH}_{3}\right), 1.33-1.28(\mathrm{~m}, 1 \mathrm{H}), 1.24(\mathrm{~m}, 6 \mathrm{H}, 2$ $\left.\mathrm{CH}_{3}\right), 1.22\left(\mathrm{~m}, 6 \mathrm{H}, 2 \mathrm{CH}_{3}\right), 1.15-1.02(\mathrm{~m}, 1 \mathrm{H}), 0.17(\mathrm{~s}, 9 \mathrm{H}, 3$ $\left.\mathrm{CH}_{3}\right)$ ppm.

(4R,5S)-4-Dimethoxymethyl-5-isopropyl-2-methyl Cyclohex-2-enone (16): This compound was prepared according to the procedure described by Gennari in $45 \%$ yield over three steps $(4.5 \mathrm{~g}) .[\alpha]_{\mathrm{D}}^{20}=$ $+158.0(c=1.07$, AcOEt $)$ \{ref. ${ }^{[9 b}[a]_{\mathrm{D}}^{20}=+146.4(c=0.98$, AcOEt) $\}.{ }^{1} \mathrm{H}$ NMR $\left(270 \mathrm{MHz}, \mathrm{CDCl}_{3}\right): \delta=6.80(\mathrm{~d}, J=2.0 \mathrm{~Hz}, 1$ $\mathrm{H}), 4.49(\mathrm{~d}, J=4.6 \mathrm{~Hz}, 1 \mathrm{H}), 3.48\left(\mathrm{~s}, 6 \mathrm{H}, 2 \mathrm{CH}_{3}\right), 2.72-2.62(\mathrm{~m}$, $1 \mathrm{H}), 2.58(\mathrm{dd}, J=16.5,4.6 \mathrm{~Hz}, 1 \mathrm{H}), 2.32(\mathrm{dd}, J=16.5,8.9 \mathrm{~Hz}$, $1 \mathrm{H}), 2.10-2.04(\mathrm{~m}, 1 \mathrm{H}), 1.90-1.74(\mathrm{~m}, 1 \mathrm{H}), 1.74\left(\mathrm{~s}, 3 \mathrm{H}, \mathrm{CH}_{3}\right)$, $0.95\left(\mathrm{~d}, J=6.6 \mathrm{~Hz}, 3 \mathrm{H}, \mathrm{CH}_{3}\right), 0.91\left(\mathrm{~d}, J=6.6 \mathrm{~Hz}, 3 \mathrm{H}, \mathrm{CH}_{3}\right) .{ }^{13} \mathrm{C}$ NMR (67.5 MHz, $\left.\mathrm{CDCl}_{3}\right): \delta=199.9(\mathrm{C}), 143.8(\mathrm{C}), 136.0(\mathrm{CH})$, $105.4(\mathrm{CH}), 55.9\left(\mathrm{CH}_{3}\right), 54.6\left(\mathrm{CH}_{3}\right), 42.3(\mathrm{CH}), 41.3(\mathrm{CH}), 37.2$ $\left(\mathrm{CH}_{2}\right), 28.2(\mathrm{CH}), 20.9\left(\mathrm{CH}_{3}\right), 17.7\left(\mathrm{CH}_{3}\right), 15.8\left(\mathrm{CH}_{3}\right)$ ppm. IR (film): $\tilde{v}=2960,2832,1690,1465,1373,1076 \mathrm{~cm}^{-1}$. MS (GC, CI, $\left.\mathrm{CH}_{4}\right): m / z=227[\mathrm{M}+\mathrm{H}]^{+} \cdot \mathrm{C}_{13} \mathrm{H}_{22} \mathrm{O}_{3}$ (226.32): calcd. $\mathrm{C} 68.99, \mathrm{H}$ 9.80; found C 69.11, H 9.84 .

$(1 R, 4 R, 5 R)-5$-Isopropyl-4-\{[(tert-butyldimethylsilyl)oxy|methyl\}-2methylcyclohex-2-enyl Diisopropylcarbamate (17): To a solution of $\mathrm{LAH}$ ( $1 \mathrm{~m}$ in $\mathrm{Et}_{2} \mathrm{O}, 11 \mathrm{~mL}, 11 \mathrm{mmol}, 0.5$ equiv.) at $-78^{\circ} \mathrm{C}$ was slowly added a solution of enone 16 ( $5 \mathrm{~g}, 22 \mathrm{mmol})$ in $\mathrm{Et}_{2} \mathrm{O}$ $(16 \mathrm{~mL})$. After $30 \mathrm{~min} \mathrm{H}_{2} \mathrm{O}(900 \mu \mathrm{L}, 50 \mathrm{mmol}), \mathrm{NaOH}(15 \% \mathrm{sol}$, $900 \mu \mathrm{L}, 3.38 \mathrm{mmol})$ and more $\mathrm{H}_{2} \mathrm{O}(2.6 \mathrm{~mL}, 142.3 \mathrm{mmol})$ were successively added to the mixture. The mixture was stirred until a white precipitate appeared; the solution was dried with $\mathrm{MgSO}_{4}$ and filtered off, and the solvent was removed under reduced pressure. The crude residue was purified by chromatography on silica gel (cyclohexane/AcOEt, 80:20) to deliver $(1 R, 4 R, 5 R)$-4-dimethoxymethyl-5-isopropyl-2-methyl cyclohex-2-enol (4.6 g, 91\%, 84\% de). $[\alpha]_{\mathrm{D}}^{20}=+112.0\left(c=1.8, \mathrm{CHCl}_{3}\right) .{ }^{1} \mathrm{H} \mathrm{NMR}\left(270 \mathrm{MHz}, \mathrm{CDCl}_{3}\right)$ : $\delta=5.54(\mathrm{~s}, 1 \mathrm{H}), 4.26(\mathrm{~d}, J=4.0 \mathrm{~Hz}, 1 \mathrm{H}), 4.13-4.09(\mathrm{~m}, 1 \mathrm{H})$, $3.40\left(\mathrm{~s}, 3 \mathrm{H}, \mathrm{CH}_{3}\right), 3.39\left(\mathrm{~s}, 3 \mathrm{H}, \mathrm{CH}_{3}\right), 2.34-2.40(\mathrm{~m}, 1 \mathrm{H}), 2.03-$ $1.91(\mathrm{~m}, 2 \mathrm{H}), 1.77$ (s, $\left.3 \mathrm{H}, \mathrm{CH}_{3}\right), 1.64-1.54(\mathrm{~m}, 1 \mathrm{H}), 1.61-1.45$ $(\mathrm{m}, 1 \mathrm{H}, \mathrm{OH}), 1.35-1.23(\mathrm{td}, J=11.8,9.2 \mathrm{~Hz}, 1 \mathrm{H}), 0.95(\mathrm{~d}, J=$ $\left.6.9 \mathrm{~Hz}, 3 \mathrm{H}, \mathrm{CH}_{3}\right), 0.84\left(\mathrm{~d}, J=6.9 \mathrm{~Hz}, 3 \mathrm{H}, \mathrm{CH}_{3}\right) \mathrm{ppm} .{ }^{13} \mathrm{C} \mathrm{NMR}$ $\left(67.5 \mathrm{MHz}, \mathrm{CDCl}_{3}\right): \delta=137.7(\mathrm{C}), 123.2(\mathrm{CH}), 106.7(\mathrm{CH}), 70.6$ $(\mathrm{CH}), 56.3\left(\mathrm{CH}_{3}\right), 55.4\left(\mathrm{CH}_{3}\right), 42.1(\mathrm{CH}), 39.4(\mathrm{CH}), 31.8\left(\mathrm{CH}_{2}\right)$, $27.1\left(\mathrm{CH}_{3}\right), 21.5(\mathrm{CH}), 19.1\left(\mathrm{CH}_{3}\right), 16.4\left(\mathrm{CH}_{3}\right) \mathrm{ppm}$. IR (film): $\tilde{v}$ $=3442,2940,1670,1450,1122,1061 \mathrm{~cm}^{-1}$. MS (GC, EI): $\mathrm{m} / z=$ 
228. $\mathrm{C}_{13} \mathrm{H}_{24} \mathrm{O}_{3}$ (228.33): calcd. C 68.38, $\mathrm{H} \mathrm{10.59;} \mathrm{found} \mathrm{C} 68.47, \mathrm{H}$ 10.65 .

To a suspension of $\mathrm{KH}$ ( $30 \%$ in oil, $1.76 \mathrm{~g}, 44 \mathrm{mmol}, 1.5$ equiv.) in THF $(20 \mathrm{~mL})$ at $0{ }^{\circ} \mathrm{C}$ was slowly added a solution of $(1 R, 4 R, 5 R)-$ 4-dimethoxymethyl-5-isopropyl-2-methyl cyclohex-2-enol $(2.0 \mathrm{~g}$, $8.8 \mathrm{mmol})$ in THF $(10 \mathrm{~mL})$. The mixture was stirred for $30 \mathrm{~min}$ at $20^{\circ} \mathrm{C}$ and then diisopropylcarbamoyl chloride $(1.72 \mathrm{~g}, 10.5 \mathrm{mmol}$, 1.2 equiv.) was added. After stirring for $30 \mathrm{~min}$ at $20^{\circ} \mathrm{C}$, the mixture was diluted at $0{ }^{\circ} \mathrm{C}$ with $\mathrm{H}_{2} \mathrm{O}(5 \mathrm{~mL})$ and an aqueous saturated $\mathrm{NH}_{4} \mathrm{Cl}$ solution $(25 \mathrm{~mL})$. After extraction with $\mathrm{Et}_{2} \mathrm{O}$, the combined organic layers were washed with brine, dried with $\mathrm{MgSO}_{4}$ and filtered off. After the solvent was removed under reduced pressure, the crude residue was purified by chromatography on silica gel (cyclohexane/AcOEt, 80:20) to deliver $(1 R, 4 R, 5 R)$-5-isopropyl-4-(dimethoxymethyl)-2-methylcyclohex-2-enyl diisopropylcarbamate (3 g, 96\%). ${ }^{1} \mathrm{H}$ NMR (400 MHz, $\left.\mathrm{CDCl}_{3}\right): \delta=5.57$ (m sharp, $\left.1 \mathrm{H}\right)$, $5.26(\mathrm{~m}, 1 \mathrm{H}), 4.23(\mathrm{~d}, J=4.1 \mathrm{~Hz}, 1 \mathrm{H}), 4.02-3.75(\mathrm{~m}, 2 \mathrm{H}), 3.38$ (s, $\left.3 \mathrm{H}, \mathrm{CH}_{3}\right), 3.36\left(\mathrm{~s}, 3 \mathrm{H}, \mathrm{CH}_{3}\right), 2.39(\mathrm{~m}, 1 \mathrm{H}), 1.96$ (ddd, $J=$ 12.4, 5.5, $2.3 \mathrm{~Hz}, 1 \mathrm{H}$ ), 1.92 (septd, $J=6.9,2.2 \mathrm{~Hz}, 1 \mathrm{H}$ ), 1.67 (s, $\left.3 \mathrm{H}, \mathrm{CH}_{3}\right), 1.61-1.54(\mathrm{~m}, 1 \mathrm{H}), 1.48$ (ddd, $J=12.4,10.5,7.8 \mathrm{~Hz}$, $1 \mathrm{H}), 1.24-1.12\left(\mathrm{~m}, 12 \mathrm{H}, 4 \mathrm{CH}_{3}\right), 0.91\left(\mathrm{~d}, J=6.9 \mathrm{~Hz}, 3 \mathrm{H}, \mathrm{CH}_{3}\right)$, $0.81\left(\mathrm{~d}, J=6.9 \mathrm{~Hz}, 3 \mathrm{H}, \mathrm{CH}_{3}\right) \mathrm{ppm} .{ }^{13} \mathrm{C} \mathrm{NMR}\left(100 \mathrm{MHz}, \mathrm{CDCl}_{3}\right)$ : $\delta=155.7(\mathrm{C}), 135.8(\mathrm{C}), 124.7(\mathrm{CH}), 106.8(\mathrm{CH}), 72.5(\mathrm{CH}), 56.3$ $\left(\mathrm{CH}_{3}\right), 55.2\left(\mathrm{CH}_{3}\right), 46.2(\mathrm{CH}), 45.8(\mathrm{CH}), 41.9(\mathrm{CH}), 39.3(\mathrm{CH})$, $28.4\left(\mathrm{CH}_{2}\right), 27.5(\mathrm{CH}), 21.8\left(\mathrm{CH}_{3}\right), 21.4\left(2 \mathrm{CH}_{3}\right), 21.0\left(2 \mathrm{CH}_{3}\right)$, $20.0\left(\mathrm{CH}_{3}\right), 17.5\left(\mathrm{CH}_{3}\right) \mathrm{ppm}$.

To a solution of $(1 R, 4 R, 5 R)$-5-isopropyl-4-(dimethoxymethyl)-2methylcyclohex-2-enyl diisopropylcarbamate $(3.7 \mathrm{~g}, 10.4 \mathrm{mmol})$ in THF $(10 \mathrm{~mL})$ and $\mathrm{H}_{2} \mathrm{O}(10 \mathrm{~mL})$ at $0{ }^{\circ} \mathrm{C}$ was slowly added formic acid ( $40 \mathrm{~mL}, 1 \mathrm{~mol}, 10$ equiv.). After stirring for $2 \mathrm{~h}$ at $20^{\circ} \mathrm{C}$, the mixture was diluted at $0{ }^{\circ} \mathrm{C}$ with $\mathrm{Et}_{2} \mathrm{O}(40 \mathrm{~mL})$ and $\mathrm{NaOH}(10 \mathrm{M}$, $100 \mathrm{~mL}$ ). The aqueous phase was treated with $\mathrm{NaOH}$ until $\mathrm{pH} 8$ and extracted with $\mathrm{Et}_{2} \mathrm{O}$. The combined organic layers were washed with brine, dried with $\mathrm{MgSO}_{4}$ and filtered off. After the solvent was removed under reduced pressure, the crude $(1 R, 4 R, 5 R)-4$-formyl-5isopropyl-2-methylcyclohex-2-enyl diisopropylcarbamate (3.4 g) was used without purification for the next step. MS (GC, EI): $\mathrm{m} / \mathrm{z}$ $=309[\mathrm{M}]^{+}$.

To a solution of $(1 R, 4 R, 5 R)$-4-formyl-5-isopropyl-2-methylcyclohex-2-enyl diisopropylcarbamate $(3.2 \mathrm{~g}, 10.4 \mathrm{mmol})$ in EtOH at $0{ }^{\circ} \mathrm{C}$ was added $\mathrm{NaBH}_{4}(100 \mathrm{mg}, 2.6 \mathrm{mmol}, 0.25$ equiv. $)$. After $20 \mathrm{~min}$, the mixture was diluted with $\mathrm{Et}_{2} \mathrm{O}(45 \mathrm{~mL})$ and $\mathrm{HCl}$ $(45 \mathrm{~mL})$. The aqueous phase was saturated by the addition of $\mathrm{NaCl}$ and extracted with $\mathrm{Et}_{2} \mathrm{O}$. The combined organic layers were washed with brine, dried with $\mathrm{MgSO}_{4}$ and filtered off. After the solvent was removed under reduced pressure, the crude residue was purified by chromatography on silica gel (cyclohexane/AcOEt, 80:20) to deliver $(1 R, 4 R, 5 R)$-4-(hydroxymethyl)-5-isopropyl-2-methylcyclohex-2enyl diisopropylcarbamate $(2.6 \mathrm{~g}, 80 \%$ over two steps $)$. $[a]_{\mathrm{D}}^{20}=$ $+86.0\left(c=1.29, \mathrm{CHCl}_{3}\right) .{ }^{1} \mathrm{H}$ NMR $\left(400 \mathrm{MHz}, \mathrm{CDCl}_{3}\right): \delta=5.58(\mathrm{~s}$ sharp, $1 \mathrm{H}), 5.37-5.30(\mathrm{~m}, 1 \mathrm{H}), 4.08-3.72(\mathrm{~m}, 2 \mathrm{H}), 3.66(\mathrm{dd}, J=$ $10.6,3.4 \mathrm{~Hz}, 1 \mathrm{H}), 3.52(\mathrm{dd}, J=10.6,5.5 \mathrm{~Hz}, 1 \mathrm{H}), 2.24-2.17(\mathrm{~m}$, $1 \mathrm{H}), 1.99$ (ddd, $J=12.2,5.5,2.3 \mathrm{~Hz}, 1 \mathrm{H}), 1.91$ (septd, $J=6.9$, $3.2 \mathrm{~Hz}, 1 \mathrm{H}), 1.69\left(\mathrm{~s}, 3 \mathrm{H}, \mathrm{CH}_{3}\right), 1.62$ (s large, $\left.1 \mathrm{H}, \mathrm{OH}\right), 1.55$ (dddd, $J=12.4,9.6,3.2,2.3 \mathrm{~Hz}, 1 \mathrm{H}), 1.45-1.33(\mathrm{~m}, 1 \mathrm{H}), 1.21$ (s, $\left.6 \mathrm{H}, 2 \mathrm{CH}_{3}\right), 1.19\left(\mathrm{~s}, 6 \mathrm{H}, 2 \mathrm{CH}_{3}\right), 0.94\left(\mathrm{~d}, J=6.9 \mathrm{~Hz}, 3 \mathrm{H}, \mathrm{CH}_{3}\right)$, $0.80\left(\mathrm{~d}, J=6.9 \mathrm{~Hz}, 3 \mathrm{H}, \mathrm{CH}_{3}\right) \mathrm{ppm} .{ }^{13} \mathrm{C} \mathrm{NMR}\left(100 \mathrm{MHz}, \mathrm{CDCl}_{3}\right)$ : $\delta=155.9(\mathrm{C}), 137.5(\mathrm{C}), 127.3(\mathrm{CH}), 73.2(\mathrm{CH}), 64.7\left(\mathrm{CH}_{2}\right), 46.4$ $(\mathrm{CH}), 45.7(\mathrm{CH}), 41.6(\mathrm{CH}), 39.4(\mathrm{CH}), 28.7\left(\mathrm{CH}_{2}\right), 27.7(\mathrm{CH})$, $21.9\left(\mathrm{CH}_{3}\right), 21.1\left(2 \mathrm{CH}_{3}\right), 20.7\left(2 \mathrm{CH}_{3}\right), 19.6\left(\mathrm{CH}_{3}\right), 16.8\left(\mathrm{CH}_{3}\right)$ ppm. IR (film): $\tilde{v}=3437,2965,1696,1445,1286,1158 \mathrm{~cm}^{-1}$. MS (GC, EI): $m / z=311[\mathrm{M}]^{+} . \mathrm{C}_{18} \mathrm{H}_{33} \mathrm{NO}_{3}$ (311.46): calcd. C 69.41, $\mathrm{H}$ 10.68, N 4.50; found C 69.47, H 10.72, N 4.54.
To a solution of $(1 R, 4 R, 5 R)$-4-(hydroxymethyl)-5-isopropyl-2methylcyclohex-2-enyl diisopropylcarbamate $(1.03 \mathrm{~g}, 3.3 \mathrm{mmol})$ in DMF $(10 \mathrm{~mL})$ at $20^{\circ} \mathrm{C}$ was added imidazole $(610 \mathrm{mg}, 9.9 \mathrm{mmol}$, 3 equiv.) and tert-butyldimethylsilyl chloride $(750 \mathrm{mg}, 4.9 \mathrm{mmol}$, 1.5 equiv.). After stirring for $3 \mathrm{~h}$ at $20^{\circ} \mathrm{C}$, the mixture was diluted with $\mathrm{Et}_{2} \mathrm{O}(10 \mathrm{~mL})$ and $\mathrm{H}_{2} \mathrm{O}(10 \mathrm{~mL})$. After extraction with $\mathrm{Et}_{2} \mathrm{O}$, the combined organic layers were washed with brine, dried with $\mathrm{MgSO}_{4}$ and filtered off. After the solvent was removed under reduced pressure, the crude residue was purified by chromatography on silica gel (cyclohexane/AcOEt, 100:0) to deliver title product $17(1.32 \mathrm{~g}, 94 \%) \cdot[\alpha]_{\mathrm{D}}^{20}=+89.0\left(c=1.04, \mathrm{CHCl}_{3}\right) .{ }^{1} \mathrm{H} \mathrm{NMR}$ $\left(400 \mathrm{MHz}, \mathrm{CDCl}_{3}\right): \delta=5.55-5.51(\mathrm{~m}, 1 \mathrm{H}), 5.34-5.28(\mathrm{~m}, 1 \mathrm{H})$, $4.11-3.73(\mathrm{~m}, 2 \mathrm{H}), 3.65(\mathrm{dd}, J=9.8,4.3 \mathrm{~Hz}, 1 \mathrm{H}), 3.37$ (dd, $J=$ 9.8, 7.1 Hz, 1 H), 2.21-2.13 (m, 1 H), 1.97 (dd, $J=9.6,5.5 \mathrm{~Hz}, 1$ $\mathrm{H}), 1.92$ (septd, $J=6.9,2.7 \mathrm{~Hz}, 1 \mathrm{H}), 1.68\left(\mathrm{~s}, 3 \mathrm{H}, \mathrm{CH}_{3}\right), 1.46-$ $1.34(\mathrm{~m}, 2 \mathrm{H}), 1.23\left(\mathrm{~s}, 6 \mathrm{H}, 2 \mathrm{CH}_{3}\right), 1.21\left(\mathrm{~s}, 6 \mathrm{H}, 2 \mathrm{CH}_{3}\right), 0.92(\mathrm{~d}$, $\left.J=6.9 \mathrm{~Hz}, 3 \mathrm{H}, \mathrm{CH}_{3}\right), 0.88\left(\mathrm{~s}, 9 \mathrm{H}, 3 \mathrm{CH}_{3}\right), 0.80(\mathrm{~d}, J=6.9 \mathrm{~Hz}$, $\left.3 \mathrm{H}, \mathrm{CH}_{3}\right), 0.03\left(\mathrm{~s}, 6 \mathrm{H}, 2 \mathrm{CH}_{3}\right) \mathrm{ppm} .{ }^{13} \mathrm{C} \mathrm{NMR}(100 \mathrm{MHz}$, $\left.\mathrm{CDCl}_{3}\right): \delta=155.9(\mathrm{C}), 135.2(\mathrm{C}), 128.4(\mathrm{CH}), 73.4(\mathrm{CH}), 65.8$ $\left(\mathrm{CH}_{2}\right), 46.2(2 \mathrm{CH}), 41.6(\mathrm{CH}), 40.2(\mathrm{CH}), 28.6\left(\mathrm{CH}_{2}\right), 27.7(\mathrm{CH})$, $26.2\left(3 \mathrm{CH}_{3}\right), 21.9\left(\mathrm{CH}_{3}\right), 21.8\left(2 \mathrm{CH}_{3}\right), 21.0\left(2 \mathrm{CH}_{3}\right), 19.6\left(\mathrm{CH}_{3}\right)$, $18.6(\mathrm{C}), 17.0\left(\mathrm{CH}_{3}\right),-5.0\left(2 \mathrm{CH}_{3}\right) \mathrm{ppm}$. IR (film): $\tilde{v}=2955,1696$, 1434, 1296, $1050 \mathrm{~cm}^{-1}$. MS (GC, CI, $\left.\mathrm{CH}_{4}\right): m / z=426[\mathrm{M}+\mathrm{H}]^{+}$. $\mathrm{C}_{24} \mathrm{H}_{47} \mathrm{NO}_{3} \mathrm{Si}$ (425.73): calcd. C 67.71, $\mathrm{H} \mathrm{11.13,} \mathrm{N} \mathrm{3.29;} \mathrm{found} \mathrm{C}$ 67.78, H 11.21, N 3.32 .

[3R(1R,2E),4R,5R]-3-[1-Hydroxy-3-methyl-5-(trimethylsilyl)pent-2en-4-ynyl]-2-methyl-5-isopropylcyclohex-1-enyl Diisopropylcarbamate (18a) and $[3 R(1 S, 2 E), 4 R, 5 R]-3-[1-H y d r o x y-3-m e t h y l-5-(t r i-$ methylsilyl)pent-2-en-4-ynyl]-2-methyl-5-isopropylcyclohex-1-enyl Diisopropylcarbamate (18b): To a solution of carbamate 17 (2.5 g, $5.9 \mathrm{mmol})$ and TMEDA (1.2 mL, $7.6 \mathrm{mmol}, 1.3$ equiv.) in toluene $(25 \mathrm{~mL})$ at $-85^{\circ} \mathrm{C}$ was slowly added $s \mathrm{BuLi}\left(1.27 \mathrm{M}\right.$ in $\mathrm{Et}_{2} \mathrm{O}, 6 \mathrm{~mL}$, $7.6 \mathrm{mmol}, 1.3$ equiv.). During the addition, the temperature was kept between $-85^{\circ} \mathrm{C}$ and $-80^{\circ} \mathrm{C}$. The clear reaction mixture became deep yellow. After $4 \mathrm{~h}$ deprotonation time at $-80^{\circ} \mathrm{C}$ a solution of aldehyde 13 (1.4 g, $8.2 \mathrm{mmol}, 1.4$ equiv.) in toluene $(3 \mathrm{~mL})$ was introduced. After stirring for $1.5 \mathrm{~h}$ at $-78^{\circ} \mathrm{C}$ the reaction was quenched by the addition of a $\mathrm{MeOH} / \mathrm{H}_{2} \mathrm{O} / \mathrm{HCl}(1 \mathrm{~N})(15 \mathrm{~mL} /$ $3 \mathrm{~mL} / 2 \mathrm{~mL}$ ) mixture. The temperature was warmed to $20^{\circ} \mathrm{C}$, and the mixture was extracted with $\mathrm{Et}_{2} \mathrm{O}$. The combined organic layers were washed with brine, dried with $\mathrm{MgSO}_{4}$ and filtered off. After the solvent was removed under reduced pressure, the crude residue was purified by chromatography on silica gel (cyclohexane/AcOEt, 90:10) to deliver title products $\mathbf{1 8 a}$ and $\mathbf{1 8 b}(3 \mathrm{~g}, 86 \%$; 18a/18b, $1: 1)$. An aliquot was purified by chromatography on silica gel (cyclohexane/AcOEt, 100:0 to 90:10) to deliver the two pure 18a and 18b isomers. Data for 18a: $[\alpha]_{\mathrm{D}}^{20}=+88.5\left(c=1.17, \mathrm{CHCl}_{3}\right) .{ }^{1} \mathrm{H}$ NMR $\left(400 \mathrm{MHz}, \mathrm{CDCl}_{3}\right): \delta=6.10(\mathrm{dq}, J=8.7,1.4 \mathrm{~Hz}, 1 \mathrm{H}), 4.65(\mathrm{~m}, 1$ H), $4.10(\mathrm{~m}, 1 \mathrm{H}), 3.91(\mathrm{dd}, J=10.5,8.7 \mathrm{~Hz}, 1 \mathrm{H}), 3.83(\mathrm{~m}, 1 \mathrm{H})$, $3.66(\mathrm{dd}, J=10.5,2.5 \mathrm{~Hz}, 1 \mathrm{H}), 2.42-2.37$ (m sharp, $1 \mathrm{H}), 2.13-$ $1.94(\mathrm{~m}, 3 \mathrm{H}), 1.79\left(\mathrm{~d}, J=1.4 \mathrm{~Hz}, 3 \mathrm{H}, \mathrm{CH}_{3}\right), 1.78-1.67(\mathrm{~m}, 2 \mathrm{H})$, $1.65\left(\mathrm{~s}, 3 \mathrm{H}, \mathrm{CH}_{3}\right), 1.34-1.16\left(\mathrm{~m}, 12 \mathrm{H}, 4 \mathrm{CH}_{3}\right), 0.92(\mathrm{~s}, 9 \mathrm{H}, 3$ $\left.\mathrm{CH}_{3}\right), 0.91-0.85(\mathrm{~m}, 6 \mathrm{H}), 0.15\left(\mathrm{~s}, 9 \mathrm{H}, 3 \mathrm{CH}_{3}\right), 0.04\left(\mathrm{~s}, 6 \mathrm{H}, 2 \mathrm{CH}_{3}\right)$ ppm. ${ }^{13} \mathrm{C} \mathrm{NMR}\left(100 \mathrm{MHz}, \mathrm{CDCl}_{3}\right): \delta=153.5$ (C), 143.3 (C), 141.2 $(\mathrm{CH}), 120.1(\mathrm{C}), 118.6(\mathrm{C}), 108.3(\mathrm{C}), 90.8(\mathrm{C}), 68.3(\mathrm{CH}), 65.0$ $\left(\mathrm{CH}_{2}\right), 46.6(\mathrm{CH}), 46.5(\mathrm{CH}), 45.7(\mathrm{CH}), 39.9(\mathrm{CH}), 39.6(\mathrm{CH})$, $27.6(\mathrm{CH}), 26.7\left(\mathrm{CH}_{2}\right), 26.0\left(3 \mathrm{CH}_{3}\right), 21.7\left(2 \mathrm{CH}_{3}\right), 20.7\left(2 \mathrm{CH}_{3}\right)$, $20.6\left(\mathrm{CH}_{3}\right), 18.3(\mathrm{C}), 17.8\left(\mathrm{CH}_{3}\right), 17.6\left(\mathrm{CH}_{3}\right), 15.2\left(\mathrm{CH}_{3}\right), 0.2(3$ $\left.\mathrm{CH}_{3}\right),-5.2\left(\mathrm{CH}_{3}\right),-5.4\left(\mathrm{CH}_{3}\right) \mathrm{ppm}$. IR (film): $\tilde{v}=3442,2955,2146$, $1685,1440,1286,1086 \mathrm{~cm}^{-1}$. MS (GC, CI, $\left.\mathrm{CH}_{4}\right): \mathrm{m} / \mathrm{z}=593[\mathrm{M}+$ $\mathrm{H}]^{+} . \mathrm{C}_{33} \mathrm{H}_{61} \mathrm{NO}_{4} \mathrm{Si}_{2}$ (592.02): calcd. C 66.95, H 10.39, N 2.37; found C 67.10, H 10.51, N 2.41. Data for 18b: $[\alpha]_{\mathrm{D}}^{20}=+25.5(c=$ $\left.0.99, \mathrm{CHCl}_{3}\right) .{ }^{1} \mathrm{H}$ NMR (400 MHz, $\left.\mathrm{CDCl}_{3}\right): \delta=5.94(\mathrm{dq}, J=8.2$, 
$1.4 \mathrm{~Hz}, 1 \mathrm{H}), 4.66$ (ddd, $J=10.5,8.2,3.4 \mathrm{~Hz}, 1 \mathrm{H}), 4.15(\mathrm{~m}, 1 \mathrm{H})$, $3.85(\mathrm{dd}, J=10.5,4.6 \mathrm{~Hz}, 1 \mathrm{H}), 3.80(\mathrm{~m}, 1 \mathrm{H}), 3.70(\mathrm{t}, J=10.5 \mathrm{~Hz}$, $1 \mathrm{H}), 3.24(\mathrm{~d}, J=10.5 \mathrm{~Hz}, 1 \mathrm{H}, \mathrm{OH}), 2.61(\mathrm{~d}, J=3.4 \mathrm{~Hz}, 1 \mathrm{H})$, $2.28(\mathrm{~m}, 1 \mathrm{H}), 2.04-1.94(\mathrm{~m}, 1 \mathrm{H}), 1.94-1.76(\mathrm{~m}, 3 \mathrm{H}), 1.84(\mathrm{~d}, J$ $\left.=1.4 \mathrm{~Hz}, 3 \mathrm{H}, \mathrm{CH}_{3}\right), 1.63\left(\mathrm{~s}, 3 \mathrm{H}, \mathrm{CH}_{3}\right), 1.33-1.19(\mathrm{~m}, 12 \mathrm{H}, 4$ $\left.\mathrm{CH}_{3}\right), 0.88\left(\mathrm{~s}, 9 \mathrm{H}, 3 \mathrm{CH}_{3}\right), 0.85\left(\mathrm{~d}, J=6.9 \mathrm{~Hz}, 3 \mathrm{H}, \mathrm{CH}_{3}\right), 0.78$ $\left(\mathrm{d}, J=6.9 \mathrm{~Hz}, 3 \mathrm{H}, \mathrm{CH}_{3}\right), 0.16\left(\mathrm{~s}, 9 \mathrm{H}, 3 \mathrm{CH}_{3}\right), 0.05(\mathrm{~s}, 6 \mathrm{H}, 2$ $\left.\mathrm{CH}_{3}\right) \mathrm{ppm} .{ }^{13} \mathrm{C} \mathrm{NMR}\left(100 \mathrm{MHz}, \mathrm{CDCl}_{3}\right): \delta=153.9(\mathrm{C}), 145.7(\mathrm{C})$, $143.4(\mathrm{CH}), 119.2(\mathrm{C}), 117.1(\mathrm{C}), 108.5(\mathrm{C}), 90.5(\mathrm{C}), 67.1(\mathrm{CH})$, $63.1\left(\mathrm{CH}_{2}\right), 47.2(\mathrm{CH}), 47.0(\mathrm{CH}), 45.6(\mathrm{CH}), 41.7(\mathrm{CH}), 36.8(\mathrm{CH})$, $27.0(\mathrm{CH}), 26.6\left(\mathrm{CH}_{2}\right), 26.1\left(3 \mathrm{CH}_{3}\right), 21.7\left(2 \mathrm{CH}_{3}\right), 20.9\left(\mathrm{CH}_{3}\right)$, $20.7\left(2 \mathrm{CH}_{3}\right), 18.5(\mathrm{C}), 17.8\left(\mathrm{CH}_{3}\right), 17.2\left(\mathrm{CH}_{3}\right), 14.5\left(\mathrm{CH}_{3}\right), 0.21(3$ $\left.\mathrm{CH}_{3}\right),-5.1\left(\mathrm{CH}_{3}\right),-5.3\left(\mathrm{CH}_{3}\right) \mathrm{ppm}$. IR (film): $\tilde{v}=3442,2955,2146$, $1685,1440,1286,1086 \mathrm{~cm}^{-1}$. MS (GC, CI, $\left.\mathrm{CH}_{4}\right): \mathrm{m} / z=593[\mathrm{M}+$ $\mathrm{H}]^{+} . \mathrm{C}_{33} \mathrm{H}_{61} \mathrm{NO}_{4} \mathrm{Si}_{2}$ (592.02): calcd. C 66.95, H 10.39, N 2.37; found $\mathrm{C} 67.05, \mathrm{H} 10.44, \mathrm{~N} 245$.

(R)-Mandelate Ester of 18a: ${ }^{1} \mathrm{H}$ NMR (400 $\left.\mathrm{MHz}, \mathrm{CDCl}_{3}\right): \delta=$ $7.39-7.28(\mathrm{~m}, 5 \mathrm{H}), 5.94(\mathrm{dq}, J=9.6,1.4 \mathrm{~Hz}, 1 \mathrm{H}), 5.87(\mathrm{dd}, J=$ $10.1,2.8 \mathrm{~Hz}, 1 \mathrm{H}), 4.62(\mathrm{~s}, 1 \mathrm{H}), 4.1-3.75(\mathrm{~m}, 2 \mathrm{H}), 3.69$ (dd, $J=$ 11.0, $6.4 \mathrm{~Hz}, 1 \mathrm{H}), 3.48(\mathrm{dd}, J=11.0,9.0 \mathrm{~Hz}, 1 \mathrm{H}), 3.34(\mathrm{~s}, 3 \mathrm{H}$, $\left.\mathrm{CH}_{3}\right), 2.50(\mathrm{~m}, 1 \mathrm{H}), 2.05-1.80(\mathrm{~m}, 3 \mathrm{H}), 1.94(\mathrm{~d}, J=1.4 \mathrm{~Hz}, 3 \mathrm{H}$, $\left.\mathrm{CH}_{3}\right), 1.75-1.60(\mathrm{~m}, 2 \mathrm{H}), 1.37\left(\mathrm{~s}, 3 \mathrm{H}, \mathrm{CH}_{3}\right), 1.25(\mathrm{~m}, 12 \mathrm{H}, 4$ $\left.\mathrm{CH}_{3}\right), 0.81\left(\mathrm{~s}, 9 \mathrm{H}, 3 \mathrm{CH}_{3}\right), 0.79\left(\mathrm{~d}, J=6.4 \mathrm{~Hz}, 3 \mathrm{H}, \mathrm{CH}_{3}\right), 0.76$ $\left(\mathrm{d}, J=6.4 \mathrm{~Hz}, 3 \mathrm{H}, \mathrm{CH}_{3}\right), 0.17\left(\mathrm{~s}, 9 \mathrm{H}, 3 \mathrm{CH}_{3}\right), 0.02\left(\mathrm{~s}, 3 \mathrm{H}, \mathrm{CH}_{3}\right)$, $0.01\left(\mathrm{~s}, 3 \mathrm{H}, \mathrm{CH}_{3}\right) \mathrm{ppm}$.

(S)-Mandelate Ester of 18a: ${ }^{1} \mathrm{H}$ NMR $\left(400 \mathrm{MHz}, \mathrm{CDCl}_{3}\right): \delta=$ $7.39-7.28(\mathrm{~m}, 5 \mathrm{H}), 5.85(\mathrm{dd}, J=9.6,3.2 \mathrm{~Hz}, 1 \mathrm{H}), 5.71(\mathrm{dq}, J=$ 9.6, $2.8 \mathrm{~Hz}, 1 \mathrm{H}), 4.68(\mathrm{~s}, 1 \mathrm{H}), 4.1-3.8(\mathrm{~m}, 2 \mathrm{H}), 3.75(\mathrm{dd}, J=$ 10.6, $6.4 \mathrm{~Hz}, 1 \mathrm{H}), 3.53(\mathrm{dd}, J=10.6,9.2 \mathrm{~Hz}, 1 \mathrm{H}), 3.39(\mathrm{~s}, 3 \mathrm{H}$, $\left.\mathrm{CH}_{3}\right), 2.70(\mathrm{~m}, 1 \mathrm{H}), 2.05-1.90(\mathrm{~m}, 3 \mathrm{H}), 1.82(\mathrm{~d}, J=1.8 \mathrm{~Hz}, 3 \mathrm{H}$, $\left.\mathrm{CH}_{3}\right), 1.80-1.60(\mathrm{~m}, 2 \mathrm{H}), 1.54\left(\mathrm{~s}, 3 \mathrm{H}, \mathrm{CH}_{3}\right), 1.25(\mathrm{~m}, 12 \mathrm{H}, 4$ $\left.\mathrm{CH}_{3}\right), 0.84\left(\mathrm{~s}, 9 \mathrm{H}, 3 \mathrm{CH}_{3}\right), 0.81\left(\mathrm{~d}, J=5.0 \mathrm{~Hz}, 3 \mathrm{H}, \mathrm{CH}_{3}\right), 0.80$ $\left(\mathrm{d}, J=5.0 \mathrm{~Hz}, 3 \mathrm{H}, \mathrm{CH}_{3}\right), 0.15\left(\mathrm{~s}, 9 \mathrm{H}, 3 \mathrm{CH}_{3}\right), 0.02(\mathrm{~s}, 6 \mathrm{H}, 2$ $\left.\mathrm{CH}_{3}\right) \mathrm{ppm}$.

(R)-Mandelate Ester of 18b: ${ }^{1} \mathrm{H} \mathrm{NMR}\left(400 \mathrm{MHz}, \mathrm{CDCl}_{3}\right): \delta=$ $7.45-7.28(\mathrm{~m}, 5 \mathrm{H}), 5.77(\mathrm{~d}, J=3.7 \mathrm{~Hz}, 1 \mathrm{H}), 5.65(\mathrm{dq}, J=8.7$, $1.4 \mathrm{~Hz}, 1 \mathrm{H}), 4.75(\mathrm{~s}, 1 \mathrm{H}), 4.1-3.80(\mathrm{~m}, 2 \mathrm{H}), 3.74$ (dd, $J=10.6$, $5.5 \mathrm{~Hz}, 1 \mathrm{H}), 3.49(\mathrm{t}, J=10.5 \mathrm{~Hz}, 1 \mathrm{H}), 3.42\left(\mathrm{~s}, 3 \mathrm{H}, \mathrm{CH}_{3}\right), 2.69$ $(\mathrm{m}, 1 \mathrm{H}), 2.10-1.80(\mathrm{~m}, 3 \mathrm{H}), 1.75\left(\mathrm{~d}, J=1.4 \mathrm{~Hz}, 3 \mathrm{H}, \mathrm{CH}_{3}\right), 1.70$ $1.65(\mathrm{~m}, 2 \mathrm{H}), 1.65\left(\mathrm{~s}, 3 \mathrm{H}, \mathrm{CH}_{3}\right), 1.26\left(\mathrm{~m}, 12 \mathrm{H}, 4 \mathrm{CH}_{3}\right), 0.87(\mathrm{~s}$, $\left.9 \mathrm{H}, 3 \mathrm{CH}_{3}\right), 0.82\left(\mathrm{~d}, J=6.4 \mathrm{~Hz}, 3 \mathrm{H}, \mathrm{CH}_{3}\right), 0.82(\mathrm{~d}, J=6.4 \mathrm{~Hz}$, $\left.3 \mathrm{H}, \mathrm{CH}_{3}\right), 0.14\left(\mathrm{~s}, 9 \mathrm{H}, 3 \mathrm{CH}_{3}\right), 0.03\left(\mathrm{~s}, 3 \mathrm{H}, \mathrm{CH}_{3}\right), 0.02(\mathrm{~s}, 3 \mathrm{H}$, $\mathrm{CH}_{3}$ ) ppm.

(S)-Mandelate Ester of 18b: ${ }^{1} \mathrm{H}$ NMR $\left(400 \mathrm{MHz}, \mathrm{CDCl}_{3}\right): \delta=$ $7.45-7.25(\mathrm{~m}, 5 \mathrm{H}), 5.88(\mathrm{dq}, J=9.1,1.4 \mathrm{~Hz}, 1 \mathrm{H}), 5.79(\mathrm{~d}, J=$ $3.7 \mathrm{~Hz}, 1 \mathrm{H}), 4.69(\mathrm{~s}, 1 \mathrm{H}), 4.1-3.80(\mathrm{~m}, 2 \mathrm{H}), 3.65(\mathrm{dd}, J=10.5$, $5.5 \mathrm{~Hz}, 1 \mathrm{H}), 3.42(\mathrm{t}, J=10.5 \mathrm{~Hz}, 1 \mathrm{H}), 3.33\left(\mathrm{~s}, 3 \mathrm{H}, \mathrm{CH}_{3}\right), 2.61$ $(\mathrm{m}, 1 \mathrm{H}), 1.92\left(\mathrm{~d}, J=1.4 \mathrm{~Hz}, 3 \mathrm{H}, \mathrm{CH}_{3}\right), 1.90-1.50(\mathrm{~m}, 5 \mathrm{H}), 1.55$ (s, $\left.3 \mathrm{H}, \mathrm{CH}_{3}\right), 1.25\left(\mathrm{~m}, 12 \mathrm{H}, 4 \mathrm{CH}_{3}\right), 0.86\left(\mathrm{~s}, 9 \mathrm{H}, 3 \mathrm{CH}_{3}\right), 0.69$ (d, $\left.J=6.9 \mathrm{~Hz}, 3 \mathrm{H}, \mathrm{CH}_{3}\right), 0.55\left(\mathrm{~d}, J=6.9 \mathrm{~Hz}, 3 \mathrm{H}, \mathrm{CH}_{3}\right), 0.17$ (s, $\left.9 \mathrm{H}, 3 \mathrm{CH}_{3}\right), 0.01$ (s, $\left.3 \mathrm{H}, \mathrm{CH}_{3}\right), 0.00\left(\mathrm{~s}, 3 \mathrm{H}, \mathrm{CH}_{3}\right) \mathrm{ppm}$.

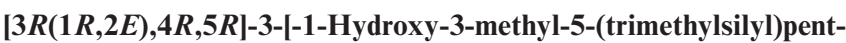
2-en-4-ynyll-5-isopropyl-4-(methoxymethyl)-2-methylcyclohex-1-enyl Diisopropylcarbamate (19a) and $(1 R, 3 \mathrm{a} R, 4 R, 7 \mathrm{a} R)-1,3,3 \mathrm{a}, 4,5,7 \mathrm{a}-$ Hexahydro-4-isopropyl-7-methyl-1-I(E)-2-methyl-4-(trimethylsilyl)but-1-en-3-ynyl|isobenzofuran-6-yl Diisopropylcarbamate (20): To a solution of 18a $(320 \mathrm{mg}, 0.55 \mathrm{mmol})$ in $\mathrm{MeOH}(7 \mathrm{~mL})$ was added Amberlyst 15 . The reaction mixture was stirred for $5.5 \mathrm{~h}$ at $20^{\circ} \mathrm{C}$, filtered through a pad of celite and the solvent was removed under reduced pressure. The crude residue was purified by chromatography on silica gel (cyclohexane/AcOEt, 80:20) to furnish compounds 19a (78 $\mathrm{mg}, 30 \%)$ and $\mathbf{2 0}(113 \mathrm{mg}, 43 \%)$ in an overall $73 \%$ yield. Data for 19a: $[\alpha]_{\mathrm{D}}^{20}=+140.2\left(c=1.2, \mathrm{CHCl}_{3}\right) .{ }^{1} \mathrm{H}$ NMR $\left(400 \mathrm{MHz}, \mathrm{CDCl}_{3}\right): \delta=6.06(\mathrm{dq}, J=8.7,1.4 \mathrm{~Hz}, 1 \mathrm{H}), 4.74(\mathrm{dd}$, $J=8.7,6.0 \mathrm{~Hz}, 1 \mathrm{H}), 4.10-4.00(\mathrm{~m}, 1 \mathrm{H}), 3.90-3.70(\mathrm{~m}, 1 \mathrm{H}), 3.82$ $(\mathrm{dd}, J=11.0,8.2 \mathrm{~Hz}, 1 \mathrm{H}), 3.71(\mathrm{dd}, J=11.0,3.4 \mathrm{~Hz}, 1 \mathrm{H}), 2.43$ $(\mathrm{m}, 1 \mathrm{H}), 2.15-1.80(\mathrm{~m}, 3 \mathrm{H}), 1.80-1.70(\mathrm{~m}, 2 \mathrm{H}), 1.82(\mathrm{~s}, 3 \mathrm{H}$, $\left.\mathrm{CH}_{3}\right), 1.64\left(\mathrm{~s}, 3 \mathrm{H}, \mathrm{CH}_{3}\right), 1.22\left(\mathrm{~m}, 12 \mathrm{H}, 4 \mathrm{CH}_{3}\right), 0.88(2 \mathrm{~d}, J=$ $\left.5.5 \mathrm{~Hz}, 3 \mathrm{H}, \mathrm{CH}_{3}\right), 0.86\left(2 \mathrm{~d}, J=5.5 \mathrm{~Hz}, 3 \mathrm{H}, \mathrm{CH}_{3}\right), 0.18(\mathrm{~s}, 9 \mathrm{H}$, $\left.3 \mathrm{CH}_{3}\right)$ ppm. ${ }^{13} \mathrm{C}$ NMR $\left(100 \mathrm{MHz}, \mathrm{CDCl}_{3}\right): \delta=153.6(\mathrm{C}), 144.0$ (C), $140.0(\mathrm{CH}), 120.3(\mathrm{C}), 119.6(\mathrm{C}), 107.9$ (C), 91.9 (C), 68.8 $(\mathrm{CH}), 64.2\left(\mathrm{CH}_{2}\right), 46.6(\mathrm{CH}), 46.5(\mathrm{CH}), 45.3(\mathrm{CH}), 39.9(\mathrm{CH})$, $39.6(\mathrm{CH}), 27.7(\mathrm{CH}), 27.0\left(\mathrm{CH}_{2}\right), 21.8\left(2 \mathrm{CH}_{3}\right), 20.8\left(\mathrm{CH}_{3}\right), 20.7$ $\left(2 \mathrm{CH}_{3}\right), 17.9\left(\mathrm{CH}_{3}\right), 17.5\left(\mathrm{CH}_{3}\right), 15.6\left(\mathrm{CH}_{3}\right), 0.2\left(3 \mathrm{CH}_{3}\right) \mathrm{ppm}$. MS $\left(\mathrm{GC}, \mathrm{CI}, \mathrm{CH}_{4}\right): \mathrm{m} / \mathrm{z}=478[\mathrm{M}+\mathrm{H}]^{+} . \mathrm{C}_{27} \mathrm{H}_{47} \mathrm{NO}_{4} \mathrm{Si}$ (477.76): calcd. C 67.88, H 9.92, N 2.93; found C 67.95, H 10.12, N 2.95. Data for 20: $[a]_{\mathrm{D}}^{20}=+28.8\left(c=1.7, \mathrm{CHCl}_{3}\right) .{ }^{1} \mathrm{H}$ NMR $\left(400 \mathrm{MHz}, \mathrm{CDCl}_{3}\right)$ : $\delta=5.90(\mathrm{dq}, J=9.6,1.4 \mathrm{~Hz}, 1 \mathrm{H}), 4.78(\mathrm{t}, J=9.6 \mathrm{~Hz}, 1 \mathrm{H}), 4.01-$ 3.89 (m, 2 H), 3.88-3.83 (m, $2 \mathrm{H}), 3.01(\mathrm{~m}, 1 \mathrm{H}), 2.32-2.23$ (m, 1 H), 2.23-2.12 (m, $1 \mathrm{H}), 1.96-1.94(\mathrm{~m}, 1 \mathrm{H}), 1.90(\mathrm{~d}, J=1.4 \mathrm{~Hz}, 3$ $\left.\mathrm{H}, \mathrm{CH}_{3}\right), 1.84$ (septd, $\left.J=6.9,3.7 \mathrm{~Hz}, 1 \mathrm{H}\right), 1.70$ (tdd, $J=11.9$, 4.6, $3.7 \mathrm{~Hz}, 1 \mathrm{H}), 1.46\left(\mathrm{~s}, 3 \mathrm{H}, \mathrm{CH}_{3}\right), 1.27\left(\mathrm{~s}, 6 \mathrm{H}, 2 \mathrm{CH}_{3}\right), 1.25$ (s, $\left.6 \mathrm{H}, 2 \mathrm{CH}_{3}\right), 0.95\left(\mathrm{~d}, J=6.9 \mathrm{~Hz}, 3 \mathrm{H}, \mathrm{CH}_{3}\right), 0.79(\mathrm{~d}, J=6.9 \mathrm{~Hz}$, $\left.3 \mathrm{H}, \mathrm{CH}_{3}\right), 0.18\left(\mathrm{~s}, 9 \mathrm{H}, 3 \mathrm{CH}_{3}\right) \mathrm{ppm} .{ }^{13} \mathrm{C} \mathrm{NMR}(100 \mathrm{MHz}$, $\left.\mathrm{CDCl}_{3}\right): \delta=153.4(\mathrm{C}), 144.6(\mathrm{C}), 136.0(\mathrm{CH}), 122.5(\mathrm{C}), 116.3(\mathrm{C})$, $108.3(\mathrm{C}), 92.5(\mathrm{C}), 75.8(\mathrm{CH}), 70.6\left(\mathrm{CH}_{2}\right), 48.2(\mathrm{CH}), 46.5(2 \mathrm{CH})$, $40.9(\mathrm{CH}), 40.4(\mathrm{CH}), 28.6(\mathrm{CH}), 26.9\left(\mathrm{CH}_{2}\right), 21.9\left(\mathrm{CH}_{3}\right), 21.8(2$ $\left.\mathrm{CH}_{3}\right), 21.0\left(2 \mathrm{CH}_{3}\right), 18.0\left(\mathrm{CH}_{3}\right), 15.9\left(\mathrm{CH}_{3}\right), 15.4\left(\mathrm{CH}_{3}\right), 0.04(3$ $\left.\mathrm{CH}_{3}\right)$ ppm. MS (GC, CI, $\left.\mathrm{CH}_{4}\right): m / z=460[\mathrm{M}+\mathrm{H}]^{+} . \mathrm{C}_{27} \mathrm{H}_{45} \mathrm{NO}_{3} \mathrm{Si}$ (459.74): calcd. C 70.54, H 9.87, N 3.05; found C 70.62, H 9.93, N 3.12 .

[3R(1S,2E),4R,5R]-3-[-1-Hydroxy-3-methyl-5-(trimethylsilyl)pent-2en-4-ynyl]-5-isopropyl-4-(methoxymethyl)-2-methylcyclohex-1-enyl Diisopropylcarbamate (19b): To a solution of $\mathbf{1 8 b}(320 \mathrm{mg}$, $0.55 \mathrm{mmol})$ in $\mathrm{MeOH}(7 \mathrm{~mL})$ was added Amberlyst 15 . The reaction mixture was stirred for $5.5 \mathrm{~h}$ at $20^{\circ} \mathrm{C}$, filtered through a pad of celite and the solvent was removed under reduced pressure. The crude residue was purified by chromatography on silica gel (cyclohexane/AcOEt, 90:10) to furnish compound 19b (257 mg, 98\%). $[\alpha]_{\mathrm{D}}^{20}=+13.6\left(c=0.97, \mathrm{CHCl}_{3}\right) .{ }^{1} \mathrm{H}$ NMR $\left(400 \mathrm{MHz}, \mathrm{CDCl}_{3}\right): \delta=$ $5.98(\mathrm{dq}, J=8.7,1.4 \mathrm{~Hz}, 1 \mathrm{H}), 4.71(\mathrm{~d}, J=8.7 \mathrm{~Hz}, 1 \mathrm{H}), 4.20$ $4.08(\mathrm{~m}, 1 \mathrm{H}), 3.92(\mathrm{dd}, J=10.9,4.6 \mathrm{~Hz}, 1 \mathrm{H}), 3.85-3.73(\mathrm{~m}, 1 \mathrm{H})$, $3.74(\mathrm{t}, J=10.9 \mathrm{~Hz}, 1 \mathrm{H}), 2.64(\mathrm{~d}, J=3.6 \mathrm{~Hz}, 1 \mathrm{H}), 2.33-2.22(\mathrm{~m}$, $1 \mathrm{H}), 2.06-1.98(\mathrm{~m}, 1 \mathrm{H}), 1.96-1.76(\mathrm{~m}, 3 \mathrm{H}), 1.85(\mathrm{~d}, J=1.4 \mathrm{~Hz}$, $\left.3 \mathrm{H}, \mathrm{CH}_{3}\right), 1.64\left(\mathrm{~s}, 3 \mathrm{H}, \mathrm{CH}_{3}\right), 1.33-1.19\left(\mathrm{~m}, 12 \mathrm{H}, 4 \mathrm{CH}_{3}\right), 0.87$ $\left(\mathrm{d}, J=6.9 \mathrm{~Hz}, 3 \mathrm{H}, \mathrm{CH}_{3}\right), 0.79\left(\mathrm{~d}, J=6.9 \mathrm{~Hz}, 3 \mathrm{H}, \mathrm{CH}_{3}\right), 0.16(\mathrm{~s}$, $\left.9 \mathrm{H}, 3 \mathrm{CH}_{3}\right)$ ppm. ${ }^{13} \mathrm{C} \mathrm{NMR}\left(100 \mathrm{MHz}, \mathrm{CDCl}_{3}\right): \delta=153.9(\mathrm{C})$, 145.7 (C), $142.6(\mathrm{CH}), 119.1$ (C), 117.7 (C), 108.3 (C), 90.9 (C), $67.6(\mathrm{CH}), 62.7\left(\mathrm{CH}_{2}\right), 47.2(\mathrm{CH}), 47.0(\mathrm{CH}), 45.7(\mathrm{CH}), 41.3(\mathrm{CH})$, $37.2(\mathrm{CH}), 27.1(\mathrm{CH}), 26.6\left(\mathrm{CH}_{2}\right), 21.7\left(2 \mathrm{CH}_{3}\right), 21.0\left(\mathrm{CH}_{3}\right), 20.8$ $\left(2 \mathrm{CH}_{3}\right), 17.7\left(\mathrm{CH}_{3}\right), 16.9\left(\mathrm{CH}_{3}\right), 14.7\left(\mathrm{CH}_{3}\right), 0.2\left(3 \mathrm{CH}_{3}\right) \mathrm{ppm} . \mathrm{MS}$ $\left(\mathrm{GC}, \mathrm{CI}, \mathrm{CH}_{4}\right): m / z=478[\mathrm{M}+\mathrm{H}]^{+} . \mathrm{C}_{27} \mathrm{H}_{47} \mathrm{NO}_{4} \mathrm{Si}$ (477.76): calcd. C 67.88, H 9.92, N 2.93; found C 67.92, H 10.04, N 2.97.

(E)-6-(tert-Butyldimethylsilyl)-3-methylhex-2-en-4-ynal (21): To a suspension of $\mathrm{Pd}(\mathrm{OAc})_{2}$ (140 mg, $0.63 \mathrm{mmol}, 0.025$ equiv.) in THF $(20 \mathrm{~mL})$ at $20^{\circ} \mathrm{C}$ was added tris(2,6-dimethoxyphenyl)phosphane ( $275 \mathrm{mg}, 1.5 \mathrm{mmol}, 0.25$ equiv.). After stirring for $15 \mathrm{~min}$ ethyl butynoate $(3.36 \mathrm{~g}, 30.0 \mathrm{mmol}$, 1 equiv.) was introduced. To the resulting clear brown mixture obtained after $5 \mathrm{~min}$ was added a solution of propargyl alcohol (1.4 g, $25.0 \mathrm{mmol}, 1$ equiv.) in THF $(5 \mathrm{~mL})$. The mixture turned dark brown. After $12 \mathrm{~h}$ at $20^{\circ} \mathrm{C}$ the mixture was concentrated under reduced pressure, and the crude residue was purified by chromatography on silica gel (cyclohexane/ AcOEt, 80:20) to deliver ethyl (E)-6-hydroxy-3-methylhex-2-en-4ynoate $(3.47 \mathrm{~g}, 83 \%) .{ }^{1} \mathrm{H}$ NMR $\left(400 \mathrm{MHz}, \mathrm{CDCl}_{3}\right): \delta=6.00(\mathrm{~s}, 1$ 
H), 4.38 (s, $2 \mathrm{H}), 4.11$ (q, $J=7.7 \mathrm{~Hz}, 2 \mathrm{H}), 2.80$ (s, $1 \mathrm{H}, \mathrm{OH}), 2.21$ (s, $\left.3 \mathrm{H}, \mathrm{CH}_{3}\right), 1.24\left(\mathrm{t}, J=7.7 \mathrm{~Hz}, 3 \mathrm{H}, \mathrm{CH}_{3}\right) \mathrm{ppm} .{ }^{13} \mathrm{C} \mathrm{NMR}$ (100 MHz, $\left.\mathrm{CDCl}_{3}\right): \delta=166.1(\mathrm{C}), 137.2(\mathrm{C}), 124.3(\mathrm{CH}), 91.8(\mathrm{C})$, $87.0(\mathrm{C}), 60.1\left(\mathrm{CH}_{2}\right), 51.4\left(\mathrm{CH}_{2}\right), 19.6\left(\mathrm{CH}_{3}\right), 14.1\left(\mathrm{CH}_{3}\right) \mathrm{ppm} . \mathrm{MS}$ $\left(\mathrm{GC}, \mathrm{CI}, \mathrm{CH}_{4}\right): m / z=168,150,139,123,111,95$. IR (film): $\tilde{v}=$ $3400,2982,1712,1616,1444,1367,1339,1258,1153,1038 \mathrm{~cm}^{-1}$.

To a solution of ethyl (E)-6-hydroxy-3-methylhex-2-en-4-ynoate $(1.24 \mathrm{~g}, 7.4 \mathrm{mmol})$ in THF $(15 \mathrm{~mL})$ was added imidazole $(0.85 \mathrm{~g}$, 12.5 mmol, 1.7 equiv.), DMAP (catalytic amount) and tert-butyldimethylsilyl chloride (1.67 g, $11.0 \mathrm{mmol}, 1.5$ equiv.). After stirring for $6 \mathrm{~h}$ at $20^{\circ} \mathrm{C}$, the reaction mixture was diluted with $\mathrm{H}_{2} \mathrm{O}$. The aqueous phase was extracted with $\mathrm{Et}_{2} \mathrm{O}$, and the combined organic layers were washed with brine, dried with $\mathrm{MgSO}_{4}$ and filtered off. After the solvent was removed under reduced pressure, the crude residue was purified by chromatography on silica gel (cyclohexane/ AcOEt, 97:3) to deliver ethyl (E)-6-(tert-butyldimethylsilyl)-3-methylhex-2-en-4-ynoate (1.88 g, 90\%). ${ }^{1} \mathrm{H}$ NMR (400 MHz, $\left.\mathrm{CDCl}_{3}\right): \delta$ $=6.02(\mathrm{~s}, 1 \mathrm{H}), 4.46(\mathrm{~s}, 2 \mathrm{H}), 4.15(\mathrm{q}, J=7.3 \mathrm{~Hz}, 2 \mathrm{H}), 2.27(\mathrm{~d}, J$ $\left.=1.4 \mathrm{~Hz}, 3 \mathrm{H}, \mathrm{CH}_{3}\right), 1.27\left(\mathrm{t}, J=7.3 \mathrm{~Hz}, 3 \mathrm{H}, \mathrm{CH}_{3}\right), 0.90(\mathrm{~s}, 9 \mathrm{H}$, $\left.3 \mathrm{CH}_{3}\right), 0.13\left(\mathrm{~s}, 6 \mathrm{H}, 2 \mathrm{CH}_{3}\right)$ ppm. ${ }^{13} \mathrm{C} \mathrm{NMR}\left(100 \mathrm{MHz}, \mathrm{CDCl}_{3}\right)$ : $\delta=166.1(\mathrm{C}), 137.6(\mathrm{C}), 124.3(\mathrm{CH}), 92.5(\mathrm{C}), 86.7(\mathrm{C}), 60.2\left(\mathrm{CH}_{2}\right)$, $52.2\left(\mathrm{CH}_{2}\right), 25.9\left(3 \mathrm{CH}_{3}\right), 19.8\left(\mathrm{CH}_{3}\right), 18.4(\mathrm{C}), 14.4\left(\mathrm{CH}_{3}\right),-4.9(2$ $\left.\mathrm{CH}_{3}\right)$ ppm. MS $\left(\mathrm{GC}, \mathrm{CI}, \mathrm{CH}_{4}\right): \mathrm{m} / z=283[\mathrm{M}+\mathrm{H}]^{+}$.

To a solution of (E)-6-(tert-butyldimethylsilyl)-3-methylhex-2-en-4ynoate $(1.67 \mathrm{~g}, 6 \mathrm{mmol})$ in $\mathrm{CH}_{2} \mathrm{Cl}_{2}(15 \mathrm{~mL})$ was added a solution of DIBAL-H at $-78^{\circ} \mathrm{C}\left(1 \mathrm{M}\right.$ in $\mathrm{CH}_{2} \mathrm{Cl}_{2}, 13 \mathrm{~mL}, 13 \mathrm{mmol}, 2.2$ equiv.). After $2 \mathrm{~h}$ at $-78^{\circ} \mathrm{C}$ the reaction mixture was quenched with ethyl acetate and then with potassium tartrate ( $24 \mathrm{mmol}, 4$ equiv.) and stirred for $1 \mathrm{~h}$. The aqueous phase was extracted with $\mathrm{CH}_{2} \mathrm{Cl}_{2}$, and the combined organic layers were washed with brine, dried with $\mathrm{MgSO}_{4}$ and filtered off. After the solvent was removed under reduced pressure (E)-6-(tert-butyldimethylsilyl)-3-methylhex-2-en-4ynol $(1.3 \mathrm{~g}, 91 \%)$ was directly used in the next step without purification. ${ }^{1} \mathrm{H}$ NMR $\left(400 \mathrm{MHz}, \mathrm{CDCl}_{3}\right): \delta=5.94(\mathrm{t}, J=6.9 \mathrm{~Hz}, 1 \mathrm{H})$, 4.39 (s, $2 \mathrm{H}), 4.19$ (d, $J=6.9 \mathrm{~Hz}, 2 \mathrm{H}), 1.91$ (s large, OH), 1.79 (s, $\left.3 \mathrm{H}, \mathrm{CH}_{3}\right), 0.89\left(\mathrm{~s}, 9 \mathrm{H}, 3 \mathrm{CH}_{3}\right), 0.10\left(\mathrm{~s}, 6 \mathrm{H}, 2 \mathrm{CH}_{3}\right) \mathrm{ppm} .{ }^{13} \mathrm{C}$ NMR (100 MHz, $\left.\mathrm{CDCl}_{3}\right): \delta=135.4(\mathrm{CH}), 120.7(\mathrm{C}), 86.9(\mathrm{C}), 86.3$ (C), $59.2\left(\mathrm{CH}_{2}\right), 52.3\left(\mathrm{CH}_{2}\right), 26.0\left(3 \mathrm{CH}_{3}\right), 18.4\left(\mathrm{CH}_{3}\right), 17.6(\mathrm{C})$, $-4.9\left(2 \mathrm{CH}_{3}\right)$ ppm. MS $\left(\mathrm{GC}, \mathrm{CI}, \mathrm{CH}_{4}\right): m / z=241[\mathrm{M}+\mathrm{H}]^{+}$.

$\operatorname{IBX}^{[24]}\left(770 \mathrm{~g}, 2.75 \mathrm{mmol}, 2.2\right.$ equiv.) was dissolved at $20^{\circ} \mathrm{C}$ in DMSO (5 mL). A solution of (E)-6-(4-methoxybenzyloxy)-3-methylhex-2-en-4-ynol (300 mg, $1.25 \mathrm{mmol})$ in DMSO $(3 \mathrm{~mL})$ was then added. After stirring for $30 \mathrm{~min}$ the aqueous phase was extracted with $\mathrm{Et}_{2} \mathrm{O}$, and the combined organic layers were washed with brine, dried with $\mathrm{MgSO}_{4}$ and filtered off. After the solvent was removed under reduced pressure, the resulting crude 21 (300 mg, $91 \%$ ) was used without further purification for the next step. ${ }^{1} \mathrm{H}$ NMR (400 MHz, $\mathrm{CDCl}_{3}$ ): $\delta=10.03(\mathrm{~d}, J=7.8 \mathrm{~Hz}, 1 \mathrm{H}), 6.17$ (d, $J=7.8 \mathrm{~Hz}, 1 \mathrm{H}), 4.49(\mathrm{~s}, 2 \mathrm{H}), 2.28\left(\mathrm{~s}, 3 \mathrm{H}, \mathrm{CH}_{3}\right), 0.91(\mathrm{~s}, 9 \mathrm{H}, 3$ $\left.\mathrm{CH}_{3}\right), 0.13$ (s, $\left.6 \mathrm{H}, 2 \mathrm{CH}_{3}\right)$ ppm. ${ }^{13} \mathrm{C} \mathrm{NMR}\left(100 \mathrm{MHz}, \mathrm{CDCl}_{3}\right): \delta$ $=190.4(\mathrm{CH}), 140.3(\mathrm{C}), 135.7(\mathrm{CH}), 97.9(\mathrm{C}), 86.1(\mathrm{C}), 52.3\left(\mathrm{CH}_{2}\right)$, $25.9\left(3 \mathrm{CH}_{3}\right), 18.5\left(\mathrm{CH}_{3}\right), 18.4(\mathrm{C}),-5.0\left(2 \mathrm{CH}_{3}\right) \mathrm{ppm}$. MS (GC, $\left.\mathrm{CI}, \mathrm{CH}_{4}\right): m / z=239[\mathrm{M}+\mathrm{H}]^{+}$.

$[3 R(1 R, 2 E), 4 R, 5 R]-3-[1-H y d r o x y-3-m e t h y l-6-($ tert-butylimethylsilyloxy)hex-2-en-4-ynyl]-2-methyl-5-isopropylcyclohex-1-enyl Diisopropylcarbamate (22a). [3R(1S,2E),4R,5R]-3-[1-Hydroxy-3methyl-6-(tert-butylimethylsilyloxy)hex-2-en-4-ynyl-2-methyl-5-isopropylcyclohex-1-enyl Diisopropylcarbamate (22b): To a solution of carbamate 17 (540 mg, $1.3 \mathrm{mmol})$ and TMEDA $(260 \mu \mathrm{L}, 1.7 \mathrm{mmol}$, 1.3 equiv.) in toluene $(8 \mathrm{~mL})$, at $-85^{\circ} \mathrm{C}$ was slowly added $s \mathrm{BuLi}$ (1.3 $\mathrm{M}$ in hexane, $1.3 \mu \mathrm{L}, 1.7 \mathrm{mmol}, 1.3$ equiv.), and the temperature was kept between $-85^{\circ} \mathrm{C}$ and $-80^{\circ} \mathrm{C}$ The clear reaction mixture became deep yellow. After $4 \mathrm{~h}$ deprotonation time at $-80^{\circ} \mathrm{C}$ a solution of aldehyde 21 ( $400 \mathrm{mg}, 1.7 \mathrm{mmol}, 1.3$ equiv.) in toluene $(5 \mathrm{~mL})$ was introduced. After stirring for $2 \mathrm{~h}$ at $-78{ }^{\circ} \mathrm{C}$ the reaction was quenched by the addition of a $\mathrm{MeOH} / \mathrm{H}_{2} \mathrm{O} / \mathrm{HCl}(1 \mathrm{~N} ; 10 \mathrm{~mL} /$ $4 \mathrm{~mL} / 2 \mathrm{~mL}$ ) mixture. The temperature was warmed to $20^{\circ} \mathrm{C}$, and the mixture was extracted with $\mathrm{Et}_{2} \mathrm{O}$. The combined organic layers were washed with brine, dried with $\mathrm{MgSO}_{4}$ and filtered off. After the solvent was removed under reduced pressure, the crude residue was purified by chromatography on silica gel (cyclohexane/AcOEt, 95:5) to deliver title product 22ab (490 mg, 57\%, 22a/22b 1:1). Data for 22a: $[a]_{\mathrm{D}}^{20}=+60.8\left(c=0.6, \mathrm{CHCl}_{3}\right) .{ }^{1} \mathrm{H} \mathrm{NMR}(400 \mathrm{MHz}$, $\left.\mathrm{CDCl}_{3}\right): \delta=5.99(\mathrm{dq}, J=8.7,1.4 \mathrm{~Hz}, 1 \mathrm{H}), 4.67(\mathrm{~m}, 1 \mathrm{H}), 4.41(\mathrm{~s}$, $2 \mathrm{H}), 4.15-4.00$ (s large, $1 \mathrm{H}), 3.90(\mathrm{dd}, J=11.0,9.1 \mathrm{~Hz}, 1 \mathrm{H})$, $3.85-3.72(\mathrm{~m}, 1 \mathrm{H}), 3.68(\mathrm{dd}, J=11.0,3.2 \mathrm{~Hz} 1 \mathrm{H}), 2.40(\mathrm{~m}, 1 \mathrm{H})$, 2.13-1.94 (m, $3 \mathrm{H}), 1.79\left(\mathrm{~d}, J=1.4 \mathrm{~Hz}, 3 \mathrm{H}, \mathrm{CH}_{3}\right), 1.77-1.67(\mathrm{~m}$, $2 \mathrm{H}), 1.65\left(\mathrm{~s}, 3 \mathrm{H}, \mathrm{CH}_{3}\right), 1.35-1.15\left(\mathrm{~m}, 12 \mathrm{H}, 4 \mathrm{CH}_{3}\right), 0.92(\mathrm{~s}, 9 \mathrm{H}$, $\left.3 \mathrm{CH}_{3}\right), 0.90$ (s, $\left.9 \mathrm{H}, 3 \mathrm{CH}_{3}\right), 0.88\left(\mathrm{~d}, J=6.9 \mathrm{~Hz}, 3 \mathrm{H}, \mathrm{CH}_{3}\right), 0.85$ (d, $\left.J=6.9 \mathrm{~Hz}, 3 \mathrm{H}, \mathrm{CH}_{3}\right), 0.05\left(\mathrm{~s}, 12 \mathrm{H}, 4 \mathrm{CH}_{3}\right) \mathrm{ppm} .{ }^{13} \mathrm{C} \mathrm{NMR}$ $\left(100 \mathrm{MHz}, \mathrm{CDCl}_{3}\right): \delta=153.5(\mathrm{C}), 143.2(\mathrm{C}), 139.9(\mathrm{CH}), 120.1$ (C), $118.2(\mathrm{C}), 87.7(\mathrm{C}), 85.2(\mathrm{C}), 68.1(\mathrm{CH}), 64.9\left(\mathrm{CH}_{2}\right), 52.4$ $\left(\mathrm{CH}_{2}\right), 47.0(\mathrm{CH}), 46.7(\mathrm{CH}), 45.6(\mathrm{CH}), 39.8(\mathrm{CH}), 39.6(\mathrm{CH})$, $27.6(\mathrm{CH}), 26.7\left(\mathrm{CH}_{2}\right), 26.0\left(3 \mathrm{CH}_{3}\right), 25.8\left(3 \mathrm{CH}_{3}\right), 21.8\left(2 \mathrm{CH}_{3}\right)$, $20.6\left(\mathrm{CH}_{3}\right), 20.5\left(2 \mathrm{CH}_{3}\right), 18.4(\mathrm{C}), 18.3(\mathrm{C}), 17.8\left(\mathrm{CH}_{3}\right), 17.7$ $\left(\mathrm{CH}_{3}\right), 15.7\left(\mathrm{CH}_{3}\right),-4.9\left(2 \mathrm{CH}_{3}\right),-5.2\left(\mathrm{CH}_{3}\right),-5.4\left(\mathrm{CH}_{3}\right)$ ppm. IR (film): $\tilde{v}=3395,2953,2925,2852,1705,1692,1458,1435,1365$, $1318,1285,1100 \mathrm{~cm}^{-1} \cdot \mathrm{C}_{37} \mathrm{H}_{69} \mathrm{NO}_{5} \mathrm{Si}_{2}$ (664.13): calcd. C 66.92, $\mathrm{H}$ 10.47, N 2.11; found C 67.11, H 10.62, N 2.27. Data for 22b: $[a]_{\mathrm{D}}^{20}=+274.4\left(c=1.05, \mathrm{CHCl}_{3}\right) .{ }^{1} \mathrm{H} \mathrm{NMR}\left(400 \mathrm{MHz}, \mathrm{CDCl}_{3}\right): \delta$ $=5.87(\mathrm{dq}, J=8.2,1.4 \mathrm{~Hz}, 1 \mathrm{H}), 4.67(\mathrm{dd}, J=8.2,4.1 \mathrm{~Hz}, 1 \mathrm{H})$, 4.40 (s, $2 \mathrm{H}), 4.18-4.05$ (s large, $1 \mathrm{H}), 3.90-3.75$ (s large, $1 \mathrm{H}), 3.85$ $(\mathrm{dd}, J=10.7,4.6 \mathrm{~Hz}, 1 \mathrm{H}), 3.70(\mathrm{t}, J=10.7 \mathrm{~Hz}, 1 \mathrm{H}), 3.47-3.3(\mathrm{~s}$ large, $1 \mathrm{H}, \mathrm{OH}), 2.62(\mathrm{~d}, J=4.1 \mathrm{~Hz}, 1 \mathrm{H}), 2.35-2.24(\mathrm{~m}, 1 \mathrm{H})$, 2.04-1.94 (m, $1 \mathrm{H}), 1.90-1.75(\mathrm{~m}, 3 \mathrm{H}), 1.83(\mathrm{~d}, J=1.4 \mathrm{~Hz}, 3 \mathrm{H}$, $\left.\mathrm{CH}_{3}\right), 1.62\left(\mathrm{~s}, 3 \mathrm{H}, \mathrm{CH}_{3}\right), 1.33-1.19\left(\mathrm{~m}, 12 \mathrm{H}, 4 \mathrm{CH}_{3}\right), 0.90(\mathrm{~s}, 9$ $\left.\mathrm{H}, 3 \mathrm{CH}_{3}\right), 0.88\left(\mathrm{~s}, 9 \mathrm{H}, 3 \mathrm{CH}_{3}\right), 0.85\left(\mathrm{~d}, J=6.9 \mathrm{~Hz}, 3 \mathrm{H}, \mathrm{CH}_{3}\right)$, $0.78\left(\mathrm{~d}, J=6.9 \mathrm{~Hz}, 3 \mathrm{H}, \mathrm{CH}_{3}\right), 0.12\left(\mathrm{~s}, 6 \mathrm{H}, 2 \mathrm{CH}_{3}\right), 0.05(\mathrm{~s}, 6 \mathrm{H}$, $\left.2 \mathrm{CH}_{3}\right)$ ppm. ${ }^{13} \mathrm{C} \mathrm{NMR}\left(100 \mathrm{MHz}, \mathrm{CDCl}_{3}\right): \delta=153.9(\mathrm{C}), 145.8$ (C), $142.2(\mathrm{CH}), 119.1(\mathrm{C}), 116.8(\mathrm{C}), 87.9(\mathrm{C}), 84.9(\mathrm{C}), 67.0(\mathrm{CH})$, $63.1\left(\mathrm{CH}_{2}\right), 52.4\left(\mathrm{CH}_{2}\right), 47.2(\mathrm{CH}), 46.9(\mathrm{CH}), 45.7(\mathrm{CH}), 41.6$ $(\mathrm{CH}), 36.8(\mathrm{CH}), 26.9(\mathrm{CH}), 26.6\left(\mathrm{CH}_{2}\right), 26.1\left(3 \mathrm{CH}_{3}\right), 26.0$ $\left.\mathrm{CH}_{3}\right), 21.7\left(2 \mathrm{CH}_{3}\right), 20.9\left(\mathrm{CH}_{3}\right), 20.7\left(\mathrm{CH}_{3}\right), 20.6\left(\mathrm{CH}_{3}\right), 18.5(2$ C), $17.8\left(\mathrm{CH}_{3}\right), 17.2\left(\mathrm{CH}_{3}\right), 14.6\left(\mathrm{CH}_{3}\right),-4.9\left(2 \mathrm{CH}_{3}\right),-5.1\left(\mathrm{CH}_{3}\right)$, $-5.3\left(\mathrm{CH}_{3}\right) \mathrm{ppm}$. IR (film): $\tilde{v}=3400,2956,2928,2856,1707,1689$, $1463,1437,1368,1315,1290,1084,835 \mathrm{~cm}^{-1} \cdot \mathrm{C}_{37} \mathrm{H}_{69} \mathrm{NO}_{5} \mathrm{Si}_{2}$ (664.13): calcd. C 66.92, H 10.47, N 2.11; found C 67.06, H 10.51, N 2.18.

Supporting Information (see footnote on the first page of this article): NMR spectra of all products.

\section{Acknowledgments}

Financial support was provided by the Institut de Recherche Pierre Fabre.

[1] a) M. D'Ambrosio, A. Guerriero, F. Pietra, Helv. Chim. Acta 1987, 70, 2019-2027; b) M. D’Ambrosio, A. Guerriero, F. Pietra, Helv. Chim. Acta 1988, 71, 964-976.

[2] S. Ketzinel, A. Rudi, M. Schleyer, Y. Benayahu, Y. Kashman, J. Nat. Prod. 1996, 59, 873-875.

[3] a) T. Lindel, P. R. Jensen, W. Fenical, B. H. Long, A. M. Casazza, J. Carboni, C. R. Fairshild, J. Am. Chem. Soc. 1997, 119, 8744 ; b) B. H. Long, J. Carboni, A. J. Wasserman, L. A. Cor- 
nell, A. M. Casazza, P. R. Jensen, T. Lindel, W. Fenical, C. R. Fairshild, Cancer Res. 1998, 58, 1111-1115.

[4] a) B. Cinel, B. O. Patrick, M. Roberge, R. J. Andersen, Tetrahedron Lett. 2000, 41, 2811-2815; b) B. Cinel, M. Roberge, H. Behrisch, L. van Ofwegen, C. B. Castro, R. J. Andersen, Org. Lett. 2000, 2, 257-260; c) M. Roberge, B. Cinel, H. J. Anderson, L. Lim, X. Jiang, L. Xu, C. M. Bigg, M. T. Kelly, R. J. Andersen, Cancer Res. 2000, 60, 5052-5058; d) R. Britton, M. Roberge, H. Berisch, R. J. Andersen, Tetrahedron Lett. 2001, 42, 2953-2956.

[5] E. Hamel, D. L. Sackett, D. Vourloumis, K. C. Nicolaou, Biochemistry 1999, 38, 5490-5498.

[6] K. C. Nicolaou, T. Ohshima, S. Hosokawa, F. L. van Delf, D. Vourloumis, J.-Y. Xu, J. Pfefferkorn, S. Kim, J. Am. Chem. Soc. 1998, 120, 8674-8680

[7] a) X.-T. Chen, B. Zhou, S. K. Bhattacharya, C. E. Gutteridge, T. R. R. Pettus, S. J. Danishefsky, Angew. Chem. Int. Ed. 1998, 37, 789-792; b) X.-T. Chen, S. K. Bhattacharya, B. Zhou, C. E. Gutteridge, T. R. R. Pettus, S. J. Danishefsky, J. Am. Chem. Soc. 1999, 121, 6563-6579.

[8] a) K. C. Nicolaou, J.-Y. Xu, S. Kim, T. Ohshima, S. Hosokawa, J. Pfefferkorn, J. Am. Chem. Soc. 1997, 119, 11353-11354; b) K. C. Nicolaou, J.-Y. Xu, S. Kim, J. Pfefferkorn, T. Ohshima, D. Vourloumis, S. Hosokawa, J. Am. Chem. Soc. 1998, 120, 8661-8673.

[9] Formal syntheses: a) N. Ritter, P. Metz, Synlett 2003, 15, 2422 2424; b) D. Castoldi, L. Caggiano, L. Panigada, O. Sharon, A. M. Costa, C. Gennari, Chem. Eur. J. 2006, 12, 51-62.

[10] Synthetic approaches: a) S. Ceccarelli, U. Piarulli, C. Gennari, Tetrahedron Lett. 1999, 40, 153-156; b) S. M. Ceccarelli, U. Piarulli, C. Gennari, Tetrahedron 2001, 57, 8531-8542; c) S. M. Ceccarelli, U. Piarulli, J. Telser, C. Gennari, Tetrahedron Lett. 2001, 42, 7421-7425; d) A. Baron, V. Caprio, J. Mann, Tetrahedron Lett. 1999, 40, 9321-9324; G. Scalabrino, X.-N. Sun, J. Mann, A. Baron, Org. Biomol. Chem. 2003, 1, 318-327; e) R. Carter, K. Hodgetts, J. Mc Kenna, P. Magnus, S. Wren, Tetrahedron 2000, 56, 4367-4382; f) P. Kim, M. H. Nantz, M. J. Kurth, M. M. Olmstead, Org. Lett. 2000, 2, 1831-1834; g) M. E. Jung, A. Huang, T. W. Johnson, Org. Lett. 2000, 2, 18351837; h) J. D. Winkler, K. J. Quinn, C. H. MacKinnon, S. D. Hiscock, E. C. McLaughlin, Org. Lett. 2003, 5, 1805-1808; i) P. Kim, M. M. Olmstead, M. H. Nantz, M. J. Kurth, Tetrahedron Lett. 2000, 41, 4029-4031; j) K. P. Kaliappan, N. Kumar, Tetrahedron Lett. 2003, 44, 379-392; k) H. Bruyere, S. Samaritani, S. Ballereau, A. Tomas, J. Royer, Synlett 2005, 1421-1424.

[11] H. C. Kolb, M. S. Van Nieuwenhze, K. B. Sharpless, Chem. Rev. 1994, 94, 2483-2547.

[12] a) D. H. R. Barton, S. W. McCombie, J. Chem. Soc. Perkin Trans. 1 1975, 1574-1585; b) D. H. R. Barton, D. O. Jang, J. Cs. Jaszberenyi, Tetrahedron Lett. 1990, 31, 3991-3994.

[13] Reduction of the O-enecarbamate functionality: a) P. Kocienski, N. J. Dixon, Synlett 1989, 52-54; b) E. De Lemos, F.-H.
Porée, A. Commerçon, J.-F. Betzer, A. Pancrazi, J. Ardisson, Angew. Chem. Int. Ed. 2007, 46, 1917-1921.

[14] For reviews, see: a) D. Hoppe, T. Hense, Angew. Chem. Int. Ed. Engl. 1997, 36, 2282-2316; b) D. Hoppe, Angew. Chem. Int. Ed. Engl. 1984, 23, 932-948; c) H. Ahlbrecht, U. Beyer, Synthesis 1999, 365-390.

[15] a) E.-U. Würthwein, D. Hoppe, J. Org. Chem. 2005, 70, 4443 4451; b) M. Özlügedik, J. Kristensen, B. Wibbeling, R. Fröhlich, D. Hoppe, Eur. J. Org. Chem. 2002, 414 427; c) O. Zschage, D. Hoppe, Tetrahedron 1992, 48, 8389-8392; d) O. Zschage, J.-R. Schwark, D. Hoppe, Angew. Chem. Int. Ed. Engl. 1990, 29, 296-298; e) D. Hoppe, O. Zschage, Angew. Chem. Int. Ed. Engl. 1989, 28, 69-71; f) T. Krämer, J.-R. Schwark, D. Hoppe, Tetrahedron Lett. 1989, 30, 7037-7040; g) O. Zschage, J.-R. Schwark, T. Krämer, D. Hoppe, Tetrahedron 1992, 48, 8377-8388. For similar sequences from alkenyl carbamates, see: h) R. Kalkofen, S. Brandau, S. Ünaldi, R. Fröhlich, D. Hoppe, Eur. J. Org. Chem. 2005, 4571-4580; i) J. Reuber, R. Fröhlich, D. Hoppe, Org. Lett. 2004, 6, 783-786; j) M. Seppi, R. Kalkofen, J. Reupohl, R. Fröhlich, D. Hoppe, Angew. Chem. Int. Ed. 2004, 43, 1423-1427.

[16] J. Reuber, R. Fröhlich, D. Hoppe, Eur. J. Org. Chem. 2005, 3017-3025.

[17] a) P. Razon, S. Dhulut, S. Bezzenine-Lafollée, J. Courtieu, A. Pancrazi, J. Ardisson, Synthesis 2005, 102-108; b) P. Razon, M.-A. N'Zoutani, S. Dhulut, S. Bezzenine-Lafollée, A. Pancrazi, J. Ardisson, Synthesis 2005, 109-121.

[18] Eleutherobin numbering will be used throughout the article.

[19] D. Hoppe, R. Hanko, A. Brönneke, F. Lichtenberg, Angew. Chem. Int. Ed. Engl. 1981, 20, 1024-1026.

[20] J. Barluenga, C. Mateos, F. Aznar, C. Valdès, J. Org. Chem. 2004, 69, 7114-7121. For preparation of the corresponding alcohol, see: B. M. Trost, M. T. Sorum, C. Chan, A. E. Harms, G. Rühter, J. Am. Chem. Soc. 1997, 119, 698-708.

[21] C9 absolute configuration was verified by ${ }^{1} \mathrm{H}$ NMR spectroscopic analysis of the $(R)$ - and $(S)$-methoxyphenylacetic esters (MPA) derived from 14a or $\mathbf{1 5}$ according to the Mosher-Trost model: a) J. M. Seco, E. Quinoà, R. Riguera, Chem. Rev. 2004, 104, 17-117; b) J. A. Dale, H. S. Mosher, J. Am. Chem. Soc. 1973, 95, 512-519; c) B. M. Trost, J. L. Belletire, S. Godleski, P. G. McDougal, J. M. Balkovec, J. J. Baldwin, M. E. Christy, G. S. Ponticello, S. L. Varga, J. P. Springer, J. Org. Chem. 1986, $51,2370-2374$.

[22] Traces $(<2 \%)$ of the C9 diastereomer of $\mathbf{1 5}$ were observed.

[23] T. Krämer, D. Hoppe, Tetrahedron Lett. 1987, 28, 5149-5152.

[24] IBX = $o$-iodoxybenzoic acid: a) D. B. Dess, J. C. Martin, J. Am. Chem. Soc. 1991, 113, 7277-7287; b) M. Frigerio, M. Santagostino, S. Sputore, G. Palmisano, J. Org. Chem. 1995, 60, $7272-7276$. 